\title{
Energy-Efficient Resource Allocation for D2D Communications in Cellular Networks
}

\author{
Tuong Duc Hoang, Student Member, IEEE, Long Bao Le, Senior Member, IEEE, and Tho Le-Ngoc, Fellow, IEEE
}

\begin{abstract}
In this paper, we study the energy-efficient resource allocation problem for device-to-device (D2D) communication underlaying cellular networks which aims to maximize the minimum weighted energy-efficiency (EE) of D2D links while guaranteeing the minimum data rates for cellular links. We first characterize the optimal power allocation of the cellular links to transform the original resource allocation problem into the joint subchannel and power allocation problem for D2D links. We then propose three resource allocation algorithms with different complexity, namely Dual-Based, Branch-and-Bound (BnB), and Relaxation-Based Rounding (RBR) algorithms. While the DualBased algorithm solves the problem by using dual decomposition method, the BnB and RBR algorithms tackle the problem by employing the relaxation approach. We establish the strong performance guarantees for the proposed algorithms through theoretical analysis. Extensive numerical studies demonstrate that the proposed algorithms achieve superior performance and significantly outperform a conventional algorithm.
\end{abstract}

Index Terms-D2D communication, cellular networks, energyefficiency, resource allocation, subchannel and power allocation

\section{INTRODUCTION}

Deployment of device-to-device communications in the wireless cellular network has been expected to significantly increase the network throughput and reduce the traffic load in the core network [1]-[7]. Efficient resource allocation algorithms play a critical role in acquiring these benefits while limiting negative impacts on the performance of existing communications between users and base-stations (BSs). In general, we can perform orthogonal or non-orthogonal spectrum sharing for D2D and cellular communication links. Orthogonal spectrum sharing assumes cellular and D2D links using distinct parts of the spectrum, and consequently the system must reserve dedicated spectral resources for D2D links. On the other hand, the non-orthogonal spectrum sharing allows D2D links to reuse the resource of cellular links in order to improve the spectrum utilization and efficiency at the costs of co-channel interference between cellular and D2D links. Since, in a cellular system, the BS has more powerful processing capacity than mobile terminals to deal with interference experienced at the receiver's side, it is more beneficial if the D2D links reuse the uplink resources of cellular links.

Copyright (C) 2015 IEEE. Personal use of this material is permitted. However, permission to use this material for any other purposes must be obtained from the IEEE by sending a request to pubs-permissions@ieee.org.

Manuscript received January 21, 2015; revised August 7, 2015; accepted September 19, 2015.

T. D. Hoang and L. B. Le are with INRS-EMT, Université du Québec, Montréal, Québec, Canada. Emails: \{tuong.hoang,long.le\}@emt.inrs.ca.

T. Le-Ngoc is with Department of Electrical and Computer Engineering, McGill University, Montréal, Québec, Canada. Emails: tho.le-ngoc@mcgill.ca
Green communication has attracted a lot of attention in recent years where maximization of EE has become an important design objective in engineering modern wireless systems [8]-[10]. In fact, development of energy-efficient resource allocation algorithms has been considered for 3GPP LTE systems [11], [12]. In general, downlink EE would be less critical than the uplink EE since BSs can have access to various energy sources while user devices are supported by energylimited batteries [13], [14].

There are some existing works considering spectrumefficient resource allocation for the D2D underlaying cellular network [15]-[19] with various objectives and system constraints. In [15], the authors develop a simple power control algorithm based on the signal to interference plus noise ratio (SINR) of the cellular link to guarantee its required performance and to maximize the sum-rate of D2D links. The authors of [16] propose a mode selection algorithm to maximize the sum-rate where they develop power control algorithms to attain the optimal solution for each mode. Power allocation for cellular and D2D links to maximize the rate of a single D2D link while guaranteeing the required rate of each cellular link is studied in [17]. In [18], the sum-rate optimization for D2D and cellular links is considered where the system with multiple D2D and multiple cellular links is studied. Finally, the work [19] develops a fair resource allocation for D2D links while assuring the required quality of service (QoS) of cellular links. In fact, to maximize the throughput or spectrum-efficiency, mobile devices would utilize large transmission powers, which may result in serious degradation of their EE. This motivates us to consider energy-efficient resource allocation for D2D communications in this current work.

There have been some initial efforts in developing energyefficient resource allocation solutions for D2D underlaying cellular networks [20]-[24]. In [20], a resource allocation solution based on non-cooperative game theory is proposed where each D2D link selfishly performs power and subchannel allocation to maximize its own EE considering the fixed resource allocation of other links. This design approach, however, may not lead to efficient utilization of the spectral resources. The authors in [21] solve the energy-efficient resource allocation problem by using the combinatorial auction game where the cellular BS acts as an auctioneer which sequentially decides the price of each resource and sells it to the set of D2D links achieving the highest utility. In this design, each D2D link is allowed to reuse the resource of only one cellular link, which may limit the achievable rates of D2D links. Moreover, a coalition game is employed to tackle the energy-efficient resource allocation problem in [22] where the authors address 
the joint mode selection and resource allocation for D2D and cellular links. Nevertheless, this work assumes that each D2D link only achieves its minimum required rate, which might not fully exploit the advantage of short-range D2D communications.

Other energy-efficient designs that aim to minimize the total power consumption are pursued in [25], [26]. However, resource allocation solutions in these papers may not fully exploit the advantages of D2D communications to achieve the optimum EE. In this paper, we study the joint subchannel and power allocation that maximizes the minimum weighted $\mathrm{EE}$ of D2D links and guarantees the minimum data rates of cellular links. Specifically, we make the following contributions.

- We formulate a general energy-efficient resource allocation problem considering multiple cellular and D2D links where each D2D link can reuse the spectral resources of multiple cellular links. This model is, therefore, more general than most models studied in the literature [15][18]. We first characterize the optimal power allocation solution for a cellular link as a function of the optimal power of the co-channel D2D link. Based on this important result, we transform the original resource allocation problem into the resource allocation problem for only D2D links.

- We propose the dual-based algorithm that solves the resource allocation problem in the dual domain. Particularly, we adopt the max-min fractional programming technique to iteratively transform the resource allocation problem into a Mixed Integer Nonlinear Programming (MINLP) problem. Then, we solve the underlying MINLP problem by using the dual decomposition approach. Theoretical analysis demonstrates that the algorithm converges to a feasible solution of the original problem. Moreover, the achieved solution is optimal if, at convergence, the duality gap of the underlying MINLP problem is zero. In addition, a distributed implementation with limited message exchange for the proposed algorithm is described, which can potentially reduce the computational burden of the BS and the system signaling overhead.

- We study the relaxation-based solution approach, which tackles the resource allocation problem by relaxing the subchannel allocation variables. In particular, we apply the branch-and-bound $(\mathrm{BnB})$ approach to branch the subchannel allocation vector space to smaller sub-spaces in which some subchannel allocation variables are determined and others are undetermined. An upper-bound is calculated by solving a max-min fractional program of the relaxed problem where all undetermined subchannel allocation variables are relaxed. In particular, we sequentially transform the relaxed problem into a convex problem and solve it by using the interior-point method until convergence. Moreover, we obtain a lower-bound of the objective value by rounding the fractional subchannel allocation solution acquired in the upper-bound calculation. Motivated by the procedure to calculate the upperbound in the $\mathrm{BnB}$ algorithm, we also propose a low- complexity Relaxation-based Rounding (RBR) algorithm. In this RBR algorithm, we first solve the relaxed problem of the original resource allocation problem for D2D links. Then, based on the obtained solution for the relaxed problem, we develop an efficient rounding procedure, which aims at minimizing the performance loss and maximize the design objective, to attain a feasible solution for the considered resource allocation problem.

- The computational complexity of the proposed algorithms is analyzed. Moreover, extensive numerical results are presented to evaluate the performance of the developed algorithms. Specifically, it is shown that the objective values achieved by the dual-based and RBR algorithms are very close to that of the optimal $\mathrm{BnB}$ algorithm. In addition, the proposed algorithms significantly outperform the conventional algorithm and the spectrumefficient resource allocation design.

The remainder of the paper is organized as follows. Section II presents the system model and problem formulation. The problem transformation is described in Section III. Sections IV and $\mathrm{V}$ develop the dual-based and relaxation-based algorithms, respectively. The computational complexity is analyzed in Section VI. Section VII presents illustrative results, followed by conclusions in Section VIII.

\section{System Model And Problem Formulation}

\section{A. System Model}

We consider uplink resource allocation scenario where cellular links share the same spectrum with multiple D2D links in a single macro-cell system. We assume that $K$ uplink cellular links in a set $\mathcal{K}=\{1, \cdots, K\}$ occupying $K$ orthogonal subchannels in the set $\mathcal{N}=\{1, \cdots, K\}$ in the considered cell. Moreover, we assume that the set $\mathcal{L}=\{1, \cdots, L\}$ of D2D links transmits data using the same set of subchannels. ${ }^{1}$ In these notations, $K=|\mathcal{K}|, L=|\mathcal{L}|$, and $N=|\mathcal{N}|$ denote the numbers of cellular links, D2D links, and subchannels, respectively, where $|A|$ denotes the cardinality of set A.

Let $h_{k l}^{n}$ denote the channel gain from the transmitter of link $l$ to the receiver of link $k$ on subchannel $n$. We assume that the subchannel allocation for cellular links has been predetermined and we are interested in allocating these subchannels to D2D links efficiently. Without loss of generality, we assume that cellular link $k$ has been allocated subchannel $k$. We introduce vector $\boldsymbol{\rho}_{l}=\left[\rho_{l}^{1}, \cdots, \rho_{l}^{K}\right]$ to describe subchannel allocation decisions for D2D link $l$ where $\rho_{l}^{k}=1$ if subchannel $k$ is allocated for D2D link $l$ and $\rho_{l}^{k}=0$, otherwise. Let $\boldsymbol{\rho}=\left[\boldsymbol{\rho}_{1}, \cdots, \boldsymbol{\rho}_{L}\right]$ denote the subchannel allocation variables for all D2D links.

We present the allocated power vectors as $\mathbf{p}=\left[\mathbf{p}_{C}, \mathbf{p}_{D}\right]$ for all the links, where $\mathbf{p}_{C}=\left[p_{C 1}^{1}, \cdots, p_{C K}^{K}\right]$ for $K$ cellular links, $\mathbf{p}_{D}=\left[\mathbf{p}_{D 1}, \cdots, \mathbf{p}_{D L}\right], \mathbf{p}_{D l}=\left[p_{D l}^{1}, \cdots, p_{D l}^{K}\right]$, for D2D links, and $p_{C k}^{k}$ and $p_{D l}^{k}$ denote the allocated transmit powers on subchannel $k$ of cellular link $k \in \mathcal{K}$ and D2D link $l \in \mathcal{L}$,

\footnotetext{
${ }^{1}$ The considered orthogonal subchannels can be sub-carriers or sub-channels in the OFDMA system or simply channels in the FDMA system.
} 
respectively. Then, the SINR achieved by cellular link $k$ on its allocated subchannel $k$ can be expressed as

$$
\Gamma_{C k}^{k}(\mathbf{p}, \boldsymbol{\rho})=\frac{p_{C k}^{k} h_{k k}^{k}}{\sigma_{k}^{k}+\sum_{l \in \mathcal{L}} \rho_{l}^{k} p_{D l}^{k} h_{k l}^{k}},
$$

where $\sum_{l \in \mathcal{L}} \rho_{l}^{k} p_{D l}^{k} h_{k l}^{k}$ represents the interference due to the D2D link using subchannel $k$ and $\sigma_{k}^{k}$ denotes the noise power on subchannel $k$. Similarly, the SINR of D2D link $l$ on subchannel $k$ can be written as

$$
\Gamma_{D l}^{k}(\mathbf{p}, \boldsymbol{\rho})=\frac{\rho_{l}^{k} p_{D l}^{k} h_{l l}^{k}}{\sigma_{l}^{k}+p_{C k}^{k} h_{l k}^{k}} .
$$

The data rates in $\mathrm{b} / \mathrm{s} / \mathrm{Hz}$ (i.e., normalized by the subchannel bandwidth) of cellular link $k \in \mathcal{K}$ on its subchannel $k$, D2D link $l \in \mathcal{L}$ on subchannel $k$, and D2D link $l$ on all the subchannels can be calculated as

$$
\begin{gathered}
R_{C k}^{k}(\mathbf{p}, \boldsymbol{\rho})=\log _{2}\left(1+\Gamma_{C k}^{k}(\mathbf{p}, \boldsymbol{\rho})\right), \\
R_{D l}^{k}(\mathbf{p}, \boldsymbol{\rho})=\log _{2}\left(1+\Gamma_{D l}^{k}(\mathbf{p}, \boldsymbol{\rho})\right),
\end{gathered}
$$

and

$$
R_{D l}(\mathbf{p}, \boldsymbol{\rho})=\sum_{k \in \mathcal{K}} \rho_{l}^{k} R_{D l}^{k}(\mathbf{p}, \boldsymbol{\rho}),
$$

respectively. We assume that the total consumed power of D2D link $l$ can be expressed as [27], [28]

$$
P_{D l}^{\text {total }}=2 P_{0}^{l}+\alpha_{l} \sum_{k \in \mathcal{K}} \rho_{l}^{k} p_{D l}^{k},
$$

where $2 P_{0}^{l}$ represents the fixed circuit power of both transmitter and receiver of D2D link $l$, and $\alpha_{l}>1$ is a factor accounting for the transmit amplifier efficiency and feeder losses.

\section{B. Problem Formulation}

In this work, we consider the resource allocation design with the following constraints. First, it is required to maintain the minimum rate of each cellular link $k$ (on its allocated subchannel $k$ ), i.e.,

$$
R_{C k}^{k}(\mathbf{p}, \boldsymbol{\rho}) \geq R_{C k}^{\min }, \forall k \in \mathcal{K} .
$$

Second, the power constraints of individual links are given as

$$
\begin{aligned}
& p_{C k}^{k} \leq P_{C k}^{\max }, \forall k \in \mathcal{K}, \\
& \sum_{k \in \mathcal{K}} \rho_{l}^{k} p_{D l}^{k} \leq P_{D l}^{\max }, \forall l \in \mathcal{L},
\end{aligned}
$$

where $P_{C k}^{\max }$ and $P_{D l}^{\max }$ are the maximum transmit powers of cellular link $k$ and D2D link $l$, respectively.

Third, the subchannel allocation variables are binary, i.e.,

$$
\rho_{l}^{k} \in\{0,1\}, \forall k \in \mathcal{K}, l \in \mathcal{L} .
$$

Finally, similar to [16]-[18], [21], [22], [25], [29], we require that each subchannel can be reused by at most one D2D link to limit the interference from D2D links to cellular links, and hence to guarantee the performance of the cellular links, i.e.,

$$
\sum_{l \in \mathcal{L}} \rho_{l}^{k} \leq 1, \forall k \in \mathcal{N}
$$

The objective of our resource allocation design is to maximize the minimum weighted EE of the D2D links. Therefore, this design can be formulated as the following energy-efficient resource allocation problem to attain the max-min fairness in weighed EE for D2D links:

$$
\begin{aligned}
\max _{\mathbf{p}, \boldsymbol{\rho}} & \min _{l \in \mathcal{L}} \frac{w_{l} R_{D l}(\mathbf{p}, \boldsymbol{\rho})}{P_{D l}^{\text {total }}} \\
\text { s.t. } & (7),(8),(9),(11),(10),
\end{aligned}
$$

where $\frac{w_{l} R_{D l}(\mathbf{p}, \boldsymbol{\rho})}{P_{D l} \text { total }}$ represents the weighted EE of the D2D links. The weight parameters $w_{l}$ can be employed to control the relative priorities among different D2D links and $\sum_{l \in \mathcal{L}} w_{l}=L$.

The resource allocation design in this paper allows each D2D link to share spectral resources with multiple cellular links but the spectral resource of each cellular link can be reused by at most one D2D link. This model allows us to (i) achieve good balance between excellent performance for D2D links and affordable interference management complexity, (ii) protect the QoS of cellular links efficiently, (iii) avoid large signaling overhead due to the Channel State Information (CSI) estimation and feedback of interfering channels among D2D links.

\section{iII. Problem Transformation}

To solve problem (12), we first describe the optimal power allocation of D2D link $l \in \mathcal{L}$ on subchannel $k \in \mathcal{N}$ in the following proposition.

Proposition 1. If $D 2 D$ link $l \in \mathcal{L}$ is allowed to reuse subchannel $k \in \mathcal{N}$ of cellular link $k$, then its power on subchannel $k, p_{D l}^{k}=\frac{1}{h_{k l}^{k}}\left(\frac{p_{C k}^{k} h_{k k}^{k}}{2^{R_{C k}^{\min }-1}}-\sigma_{k}^{k}\right) \in\left[0, P_{D l k}^{\max }\right]$, where $p_{C k}^{k}$ is the power of cellular link $k$, and $P_{D l k}^{\max }=\min \left\{P_{D l}^{\max }\right.$, $\left.\frac{1}{h_{k l}^{k}}\left(\frac{P_{C k}^{\max } h_{k k}^{k}}{2^{R_{C k}^{\min }-1}}-\sigma_{k}^{k}\right)\right\}$.

Proof. The proof is given in Appendix A.

From Proposition 1, the data rate of D2D link $l$ on subchannel $k$ given in (4) can be re-written as

$$
\begin{aligned}
& \hat{R}_{D l}^{k}\left(\mathbf{p}_{D}, \boldsymbol{\rho}\right) \\
& =\rho_{l}^{k} \log _{2}\left(1+\frac{p_{D l}^{k} h_{l l}^{k}}{\sigma_{l}^{k}+\frac{\left(2^{\left.R_{C k}^{\min }-1\right) h_{l k}^{k}}\right.}{h_{k k}^{k}}\left(\sigma_{k}^{k}+p_{D l}^{k} h_{k l}^{k}\right)}\right) .
\end{aligned}
$$

For convenience, let us define

$$
\begin{aligned}
& a_{k l} \triangleq \frac{\sigma_{l}^{k}}{h_{l l}^{k}}+\frac{\left(2^{R_{k}^{\min }}-1\right) h_{l k}^{k} \sigma_{k}^{k}}{h_{k k}^{k} h_{l l}^{k}} \\
& b_{k l} \triangleq \frac{\left(2^{R_{k}^{\min }}-1\right) h_{l k}^{k} h_{k l}^{k}}{h_{k k}^{k} h_{l l}^{k}} .
\end{aligned}
$$


Then, the data rate of D2D link $l$ on subchannel $k \in \mathcal{N}$, $\hat{R}_{D l}^{k}\left(\mathbf{p}_{D}, \boldsymbol{\rho}\right)$ in (13), and the total rate over all subchannels, $\hat{R}_{D l}\left(\mathbf{p}_{D}, \boldsymbol{\rho}\right)$ in (5), can be rewritten, respectively, as

$$
\hat{R}_{D l}^{k}\left(\mathbf{p}_{D}, \boldsymbol{\rho}\right)=\rho_{l}^{k} \log _{2}\left(1+\frac{p_{D l}^{k}}{a_{k l}+b_{k l} p_{D l}^{k}}\right),
$$

and

$$
\hat{R}_{D l}\left(\mathbf{p}_{D}, \boldsymbol{\rho}\right)=\sum_{k \in \mathcal{N}} \hat{R}_{D l}^{k}\left(\mathbf{p}_{D}, \boldsymbol{\rho}\right),
$$

where the allocated transmit power must satisfy

$$
p_{D l}^{k} \leq P_{D l k}^{\max }, \forall k \in \mathcal{N}, \forall l \in \mathcal{L} .
$$

Therefore, problem (12) is equivalent to the following

$$
\max _{\left(\mathbf{p}_{D}, \boldsymbol{\rho}\right)} \min _{l \in \mathcal{L}} \frac{w_{l} \hat{R}_{D l}\left(\mathbf{p}_{D l}, \boldsymbol{\rho}\right)}{P_{D l}^{\text {total }}\left(\mathbf{p}_{D l}, \boldsymbol{\rho}\right)}
$$$$
\text { s.t. } \quad(9),(10),(11),(18) \text {. }
$$

In order to solve problem (19), we consider the following optimization problem

$$
\max _{\mathbf{p}_{D}, \boldsymbol{\rho}} \eta\left(\zeta, \mathbf{p}_{D}, \boldsymbol{\rho}\right) \triangleq \min _{l \in \mathcal{L}}\left[w_{l} \hat{R}_{D l}\left(\mathbf{p}_{D}, \boldsymbol{\rho}\right)-\zeta P_{D l}^{\text {total }}\left(\mathbf{p}_{D}, \boldsymbol{\rho}\right)\right]
$$$$
\text { s.t. }(9),(10),(11),(18) \text {. }
$$

Suppose that $\eta^{*}(\zeta)=\eta\left(\zeta, \mathbf{p}_{D}^{*}, \boldsymbol{\rho}^{*}\right)$ where $\left(\mathbf{p}_{D}^{*}, \boldsymbol{\rho}^{*}\right)$ is the optimal solution of problem (20), and $\mathcal{D}$ denotes the set of feasible solutions of problem (19). Then, we can characterize the optimal solution of problem (20) in the following theorem, which is adopted from [30].

Theorem 1. $\eta^{*}(\zeta)$ is a decreasing function of $\zeta$. In addition, if we have

$$
\begin{aligned}
& \max _{\left(\boldsymbol{p}_{D}, \boldsymbol{\rho}\right) \in \mathcal{D}} \min _{l \in \mathcal{L}}\left[w_{l} \hat{R}_{D l}\left(\boldsymbol{p}_{D}, \boldsymbol{\rho}\right)-\zeta^{*} P_{D l}^{\text {total }}\left(\boldsymbol{p}_{D}, \boldsymbol{\rho}\right)\right] \\
& =\min _{l \in \mathcal{L}}\left[w_{l} \hat{R}_{D l}\left(\boldsymbol{p}_{D}^{*}, \boldsymbol{\rho}^{*}\right)-\zeta^{*} P_{D l}^{\text {total }}\left(\boldsymbol{p}^{*}, \boldsymbol{\rho}^{*}\right)\right]=0
\end{aligned}
$$

then $\zeta^{*}=\min _{l \in \mathcal{L}} \frac{w_{l} \hat{R}_{D l}\left(\boldsymbol{p}_{D}^{*}, \boldsymbol{\rho}^{*}\right)}{P_{D l}^{\text {total }}\left(\boldsymbol{p}_{D}^{*}, \boldsymbol{\rho}^{*}\right)}$ is the optimal solution of (19).

It is worth noting that the main theorem in [30] states the necessary and sufficient condition for $\zeta^{*}$ to be the optimal solution of the fractional programming problem. In Theorem 1 , we only present the sufficient condition for $\zeta^{*}$ to be the optimal solution of problem (19). However, the theorem of [30] requires the set of feasible solutions of the fractional programming problem to be continuous, which is not required in our theorem. Importantly, Theorem 1 allows us to transform a general max-min fractional problem (19) to a non-fractional optimization problem with the parameter $\zeta$. In addition, the optimal solution of problem (19), $\zeta^{*}$, can be found if $\eta^{*}\left(\zeta^{*}\right)=0$. Since $\eta^{*}(\zeta)$ is a decreasing function of $\zeta$, it can be seen that $\zeta^{*}$ can be indeed determined by the gradient or bisection method.

A general algorithm solving problem (19) based on the solution of problem (20) is described in Algorithm 1, which iteratively solves problem (20) for given $\zeta$ and updates $\zeta$ until convergence. Therefore, the remaining challenge is how to solve problem (20) for a given $\zeta$. In general, problem (20) is NP-hard, which implies that solving this problem optimally requires exponential complexity.

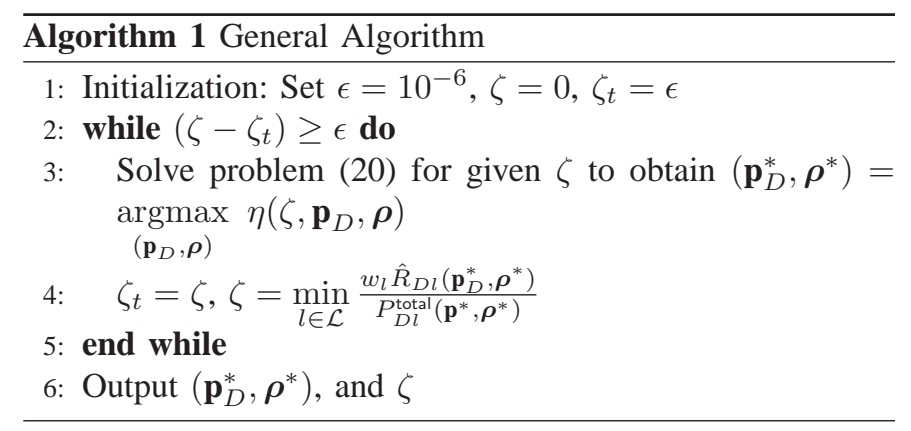

\section{Dual-Based Algorithm}

In this section, we propose a dual-based algorithm to solve problem (19). Then, we will present the distributed implementation for this algorithm.

\section{A. Algorithm Development}

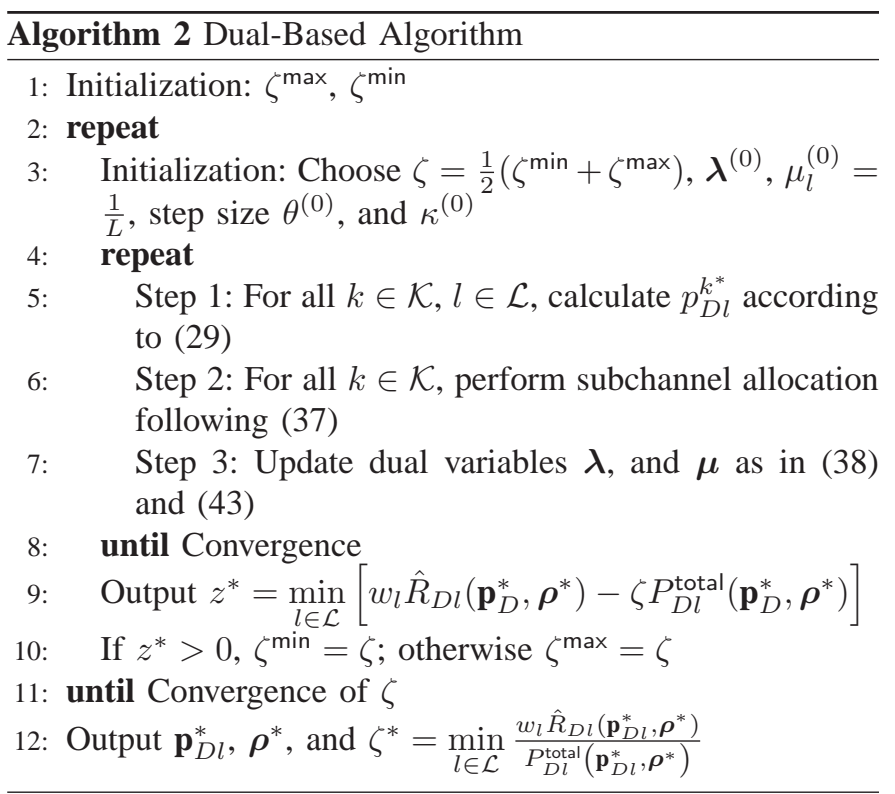

The dual-based resource allocation algorithm is summarized in Algorithm 2. The algorithm comprises two iterative loops. In the outer loop, we adopt the max-min fractional programming technique described in Algorithm 1 to attain the optimal value of $\zeta$ for problem (19). In the inner loop, we solve problem (20) for a given $\zeta$ by employing the dual decomposition method.

Algorithm 2 performs two main tasks, which are executed sequentially. Specifically, we solve problem (20) for a given $\zeta$ (lines 4-8) in the first task while we update the value of $\zeta$ based on the results of the first task by using the bisection method in the second task (line 10). In the following, we show how to solve problem (20) for a given value of $\zeta$. First, it can be observed that problem (20) is equivalent to the following problem

$$
\begin{aligned}
\max _{z, \mathbf{p}_{D}, \boldsymbol{\rho}} & z \\
\text { s.t. } & w_{l} \hat{R}_{D l}\left(\mathbf{p}_{D l}, \boldsymbol{\rho}\right)-\zeta P_{D l}^{\text {total }}\left(\mathbf{p}_{D}, \boldsymbol{\rho}\right) \geq z, \forall l \in \mathcal{L} \\
& (9),(10),(11),(18) .
\end{aligned}
$$


To tackle problem (22), we consider its Lagrangian as follows:

$$
\begin{aligned}
& L_{D}\left(\mathbf{p}_{D}, \boldsymbol{\rho}, z, \zeta, \boldsymbol{\lambda}, \boldsymbol{\mu}\right) \\
& \begin{array}{l}
=z+\sum_{l \in \mathcal{L}} \mu_{l}\left[w_{l} \hat{R}_{D l}\left(\mathbf{p}_{D}, \boldsymbol{\rho}\right)-\zeta P_{D l}^{\text {total }}\left(\mathbf{p}_{D}, \boldsymbol{\rho}\right)-z\right] \\
\quad+\sum_{l \in \mathcal{L}} \lambda_{l}\left(P_{D l}^{\max }-\sum_{k \in \mathcal{N}} \rho_{l}^{k} p_{D l}^{k}\right) \\
=z\left(1-\sum_{l \in \mathcal{L}} \mu_{l}\right)+\sum_{l \in \mathcal{L}} \mu_{l}\left[w_{l} \hat{R}_{D l}\left(\mathbf{p}_{D}, \boldsymbol{\rho}\right)-\zeta P_{D l}^{\text {total }}\left(\mathbf{p}_{D}, \boldsymbol{\rho}\right)\right] \\
\quad+\sum_{l \in \mathcal{L}} \lambda_{l}\left(P_{D l}^{\max }-\sum_{k \in \mathcal{N}} \rho_{l}^{k} p_{D l}^{k}\right),
\end{array}
\end{aligned}
$$

where $\boldsymbol{\lambda}=\left[\lambda_{1}, \cdots, \lambda_{L}\right]^{T}$ and $\boldsymbol{\mu}=\left[\mu_{1}, \cdots, \mu_{L}\right]^{T}$ represent the Lagrange multipliers. Then, the dual function can be written as

$$
\bar{L}_{D}(\zeta, \boldsymbol{\lambda}, \boldsymbol{\mu}) \triangleq \max _{z, \mathbf{p}_{D} \in \mathcal{X}, \boldsymbol{\rho} \in \mathcal{C}} L_{D}\left(\mathbf{p}_{D}, \boldsymbol{\rho}, z, \zeta, \boldsymbol{\lambda}, \boldsymbol{\mu}\right),
$$

where $\mathcal{X}=\left\{\mathbf{p}_{D} \mid p_{D l}^{k} \leq P_{D l k}^{\max }, \forall k \in \mathcal{N}, \forall l \in \mathcal{L}\right\}$, and $\mathcal{C}=$ $\left\{\boldsymbol{\rho} \mid \sum_{l \in \mathcal{L}} \rho_{l}^{k} \leq 1, \forall k \in \mathcal{N}\right.$, and $\left.\rho_{l}^{k} \in\{0,1\}, \forall k \in \mathcal{K}, l \in \mathcal{L}\right\}$. Then, the dual problem can be stated as

$$
\hat{L}_{D}(\zeta) \triangleq \min _{\boldsymbol{\lambda}, \boldsymbol{\mu} \geq 0} \bar{L}_{D}(\zeta, \boldsymbol{\lambda}, \boldsymbol{\mu})
$$

In order to solve the dual problem (25), we investigate problem (24) for the given $\boldsymbol{\lambda}$ and $\boldsymbol{\mu}$. In particular, we have

$$
\begin{aligned}
& \bar{L}_{D}(\zeta, \boldsymbol{\lambda}, \boldsymbol{\mu})=\max _{z, \mathbf{p}_{D} \in \mathcal{X}, \boldsymbol{\rho} \in \mathcal{C}} L_{D}\left(\mathbf{p}_{D}, \boldsymbol{\rho}, z, \zeta, \boldsymbol{\lambda}, \boldsymbol{\mu}\right) \\
= & \max _{z, \mathbf{p}_{D} \in \mathcal{X}, \boldsymbol{\rho} \in \mathcal{C}} \\
& \sum_{k \in \mathcal{N}} \sum_{l \in \mathcal{L}} \rho_{l}^{k}\left[\mu_{l} w_{l} \hat{R}_{D l}^{k}\left(\mathbf{p}_{D}, \boldsymbol{\rho}\right)-\left(\zeta \alpha_{l} \mu_{l}+\lambda_{l}\right) p_{D l}^{k}\right] \\
& +z\left(1-\sum_{l \in \mathcal{L}} \mu_{l}\right)+\sum_{l \in \mathcal{L}}\left(\lambda_{l} P_{D l}^{\max }-2 \zeta \mu_{l} P_{0}^{l}\right) .
\end{aligned}
$$

Note that $z$ is an uncontrolled variable in problem (26). Thus, to obtain the nontrivial optimal solution of the dual problem (25), $\sum_{l \in \mathcal{L}} \mu_{l}=1$ must hold. Moreover, problem (26) can be decomposed into $N$ individual resource allocation problems for $N$ subchannels where the resource allocation problem for subchannel $k \in \mathcal{N}$ can be stated as

$$
\begin{aligned}
& \bar{L}_{D}^{k}(\zeta, \boldsymbol{\lambda}, \boldsymbol{\mu}) \\
& =\max _{\mathbf{p}_{D} \in \mathcal{X}, \boldsymbol{\rho} \in \mathcal{C}} \sum_{l \in \mathcal{L}} \rho_{l}^{k}\left[\mu_{l} w_{l} \hat{R}_{D l}^{k}\left(\mathbf{p}_{D}, \boldsymbol{\rho}\right)-\left(\zeta \alpha_{l} \mu_{l}+\lambda_{l}\right) p_{D l}^{k}\right] .
\end{aligned}
$$

Then,

$$
\begin{aligned}
& \bar{L}_{D}(\zeta, \boldsymbol{\lambda}, \boldsymbol{\mu}) \\
& =\sum_{k \in \mathcal{N}} \bar{L}_{D}^{k}(\zeta, \boldsymbol{\lambda}, \boldsymbol{\mu})+\sum_{l \in \mathcal{L}}\left(\lambda_{l} P_{D l}^{\max }-2 \zeta \mu_{l} P_{0}^{l}\right) .
\end{aligned}
$$

We now define

$$
f_{l}^{k}\left(p_{D l}^{k}\right) \triangleq \mu_{l} w_{l} \hat{R}_{D l}^{k}\left(\mathbf{p}_{D}, \boldsymbol{\rho}\right)-\left(\zeta \alpha_{l} \mu_{l}+\lambda_{l}\right) p_{D l}^{k} .
$$

For problem (27), suppose that D2D link $l$ is allocated subchannel $k \in \mathcal{N}$ then we have

$$
p_{D l}^{k^{*}}=\underset{p_{D l}^{k} \in \mathcal{X}_{l}}{\operatorname{argmax}} f_{l}^{k}\left(p_{D l}^{k}\right) \text {. }
$$

Note that we must have $\mu_{l}>0$. This is because if $\mu_{l}=0$, we have $p_{D l}^{k *}=0, \forall k \in \mathcal{N}$, which cannot be the optimal solution of problem (26). In addition, problem (30) can be addressed by solving $\frac{\partial f_{l}^{k}\left(p_{D l}^{k}\right)}{\partial p_{D l}^{k}}=0$, where $\frac{\partial f_{l}^{k}\left(p_{D l}^{k}\right)}{\partial p_{D l}^{k}}$ is the first order derivative of $f_{l}^{k}\left(p_{D l}^{k}\right)$, which can be written as

$$
\frac{\partial f_{l}^{k}}{\partial p_{D l}^{k}}=\frac{\mu_{l} w_{l} a_{k l} \log _{2} e}{\left(a_{k l}+b_{k l} p_{D l}^{k}\right)\left[a_{k l}+\left(b_{k l}+1\right) p_{D l}^{k}\right]}-\left(\zeta \alpha_{l} \mu_{l}+\lambda_{l}\right) .
$$

Then, it can be verified that solving $\frac{\partial f_{l}^{k}\left(p_{D l}^{k}\right)}{\partial p_{D l}^{k}}=0$ is equivalent to solving $A_{k l}\left(p_{D l}^{k}\right)^{2}+2 B_{k l} p_{D l}^{k}+C_{k l}=0$ where

$$
\begin{aligned}
& A_{k l}^{d} \triangleq\left(\zeta \alpha_{l}+\frac{\lambda_{l}}{\mu_{l}}\right) b_{k l}\left(b_{k l}+1\right) \\
& B_{k l}^{d} \triangleq\left(\zeta \alpha_{l}+\frac{\lambda_{l}}{\mu_{l}}\right)\left(a_{k l} b_{k l}+0.5 a_{k l}\right) \\
& C_{k l}^{d} \triangleq\left(\zeta \alpha_{l}+\frac{\lambda_{l}}{\mu_{l}}\right) a_{k l}^{2}-w_{l} a_{k l} \log _{2} e \\
& \Delta_{k l}^{d} \triangleq\left(B_{k l}^{d}\right)^{2}-A_{k l}^{d} C_{k l}^{d} .
\end{aligned}
$$

Consequently, the optimal solution of D2D link $l$ that maximizes $f_{l}^{k}\left(p_{D l}^{k}\right)$ is given by

$$
p_{D l}^{k^{*}}=\left[\frac{-B_{k l}^{d}+\sqrt{\Delta_{k l}^{d}}}{A_{k l}^{d}}\right]_{0}^{P_{D l}^{\max }},
$$

where $[x]_{a}^{b}=b$ if $x>b,[x]_{a}^{b}=a$ if $x<a$, otherwise $[x]_{a}^{b}=x$.

In summary, by solving problem (26) we can obtain the optimal power allocation for any D2D link on subchannel $k \in \mathcal{N}$. Recall that we have assumed that each subchannel can be allocated to at most one D2D link; therefore, for all subchannels $k \in \mathcal{N}$, we have

$$
\rho_{l}^{k^{*}}= \begin{cases}1 & \text { if } l=\underset{l \in \mathcal{L}}{\operatorname{argmax}} f_{l}^{k}\left(p_{D l}^{k^{*}}\right) \\ 0 & \text { otherwise. }\end{cases}
$$

So far we have presented the resource allocation solution for given $\boldsymbol{\lambda}, \boldsymbol{\mu}$. Therefore, the remaining task is to solve problem (25), which can be completed by the sub-gradient method as described in the following. In the initial iteration $s=0$, we solve problem (24) with the initial value of $\boldsymbol{\lambda}^{(0)}$ and $\boldsymbol{\mu}^{(0)}$. Then, in iteration $s+1$, we update the dual variables $\boldsymbol{\lambda}^{(s+1)}$ and $\boldsymbol{\mu}^{(s+1)}$ based on the solution in iteration $s$, then we solve problem (24) with the updated value of $\boldsymbol{\lambda}$ and $\boldsymbol{\mu}$. The procedure to update $\lambda$ and $\boldsymbol{\mu}$ by using the sub-gradient method can be expressed as follows

$$
\lambda_{l}^{(s+1)}=\left[\lambda_{l}^{(s)}+\theta_{l}^{(s)}\left(\sum_{k \in \mathcal{N}} \rho_{l}^{k^{*}} p_{D l}^{k^{*}(s)}-P_{D l}^{\max }\right)\right]^{+}, \forall l \in \mathcal{L}
$$

$\mu_{l}^{\prime(s+1)}=\left[\mu_{l}^{(s)}-\kappa_{l}^{(s)}\left(z_{l}^{(s)}-z_{\min }^{(s)}\right)\right]^{+}, \forall l \in \mathcal{L}$, 
where $[x]^{+}=\max \{x, 0\}$, and

$$
\begin{aligned}
& z_{l}^{(s)}=w_{l} R_{D l}\left(\boldsymbol{p}_{D}^{(s)}, \boldsymbol{\rho}^{(s)}\right)-\zeta\left(2 P_{0}^{l}+\alpha_{l} \sum_{k \in \mathcal{N}} \rho_{l}^{k^{*}} p_{D l}^{k^{*}(s)}\right) \\
& z_{\min }^{(s)}=\min _{l \in \mathcal{L}}\left[w_{l} R_{D l}\left(\boldsymbol{p}_{D}^{(s)}, \boldsymbol{\rho}^{(s)}\right)-\zeta\left(2 P_{0}^{l}+\alpha_{l} \sum_{k \in \mathcal{N}} \rho_{l}^{k^{*}} p_{D l}^{k^{*}(s)}\right)\right]
\end{aligned}
$$

and $\theta_{l}^{(s)}, \kappa_{l}^{(s)}$ are step sizes, which can be chosen appropriately to ensure the convergence of the underlying iterative updates. Note that $\boldsymbol{p}_{D}^{(s)}, \boldsymbol{\rho}^{(s)}$ are, respectively, the transmit power and subchannel allocation solutions for given $\boldsymbol{\lambda}^{(s)}, \boldsymbol{\mu}^{(s)}$. Recall that $\boldsymbol{\mu}$ must satisfy the constraint $\sum_{l \in \mathcal{L}} \mu_{l}=1$. Therefore, we normalize $\boldsymbol{\mu}^{(s+1)}$ as follows:

$$
\mu_{l}^{(s+1)}=\frac{\mu_{l}^{\prime(s+1)}}{\sum_{l \in \mathcal{L}} \mu_{l}^{\prime(s+1)}}, \forall l \in \mathcal{L} .
$$

It is shown in [35] that the dual decomposition procedure converges to the optimal solution of problem (25) for appropriately chosen $\theta_{l}^{(s)}$ and $\kappa_{l}^{(s)}$. Therefore, the iterative loop in the first task of Algorithm 2 always converges to the dual solution of problem (20) for any value of $\zeta$. On the other hand, the performance achieved by Algorithm 2, which solves problem (19), is stated in the following proposition

Proposition 2. Algorithm 2 returns a feasible solution of problem (19) with $\zeta^{*}, \boldsymbol{p}_{D}^{*}, \boldsymbol{\rho}^{*} \boldsymbol{\lambda}^{*}$, and $\boldsymbol{\mu}^{*}$ at the end of its first phase. Moreover, if $\sum_{k \in \mathcal{N}} \rho_{l}^{k^{*}} p_{D l}^{k^{*}} \leq P_{D l}^{\max }, \lambda_{l}^{*}\left(P_{D}^{\max }-\right.$ $\left.\sum_{k \in \mathcal{N}} \rho_{l}^{k^{*}} p_{D l}^{k^{*}}\right)=0$, and $R_{D l}\left(\boldsymbol{p}_{D}^{*}, \boldsymbol{\rho}^{*}\right)-\zeta^{*} P_{D l}^{\text {total }}\left(\boldsymbol{p}_{D}^{*}, \boldsymbol{\rho}^{*}\right)=$ $0, \forall l \in \mathcal{L}$, this feasible solution is the optimal solution of problem (19).

Proof. The proof is provided in Appendix C.

\section{B. Distributed Implementation with Limited Message Passing}

The distributed implementation with limited message exchange to execute Algorithm 2 is now described. In this implementation, instead of performing all the necessary tasks, the $\mathrm{BS}$ assigns some to the $\mathrm{D} 2 \mathrm{D}$ links to reduce the computational burden on the BS. Due to the QoS requirements of cellular links and the strong interference coupling among wireless links, certain coordination among the BS and mobile devices via message passing deems necessary to achieve efficient spectrum sharing for D2D and cellular links. ${ }^{2}$

We can modify the procedure to update the dual-variable $\mu$, which is introduced to adjust $z_{l}^{(s)}$ in each iteration, with $\sum_{l \in \mathcal{L}} \mu_{l}=1$ as

$$
\mu_{l}^{(s+1)}=\mu_{l}^{(s)}+\kappa_{l}^{(s)},
$$

\footnotetext{
${ }^{2}$ Distributed resource allocation algorithms can also be developed by using advanced game-theory and learning techniques [32], [33]. We will explore these solution approaches in our future works.
}

where $\kappa_{l}^{(s)}>0$ if $z_{l}^{(s)}=z_{\min }^{(s)}$ and $\kappa_{l}^{(s)}<0$, otherwise, and $\kappa_{l}^{(s)}$ is chosen to satisfy $\sum_{l \in \mathcal{L}} \kappa_{l}^{(s)}=0$. A typical update of $\boldsymbol{\mu}$ with a fixed step-size can be implemented as

$$
\kappa_{l}^{(s)}=\left\{\begin{array}{l}
\kappa, \text { if } z_{l}^{(s)}=z_{\min }^{(s)} \\
\frac{-\kappa}{L-1}, \text { otherwise }
\end{array}\right.
$$

where $\kappa$ is a small value to guarantee the convergence of the updates [35]. The distributed procedure for Algorithm 2 can be described as follows.

\section{Initialization:}

Each D2D link $l$ initializes the following system parameters: $\zeta^{\max }, \zeta^{\min }, \zeta=\frac{1}{2}\left(\zeta^{\min }+\zeta^{\max }\right), \lambda_{l}^{(0)}=0, \mu_{l}^{(0)}=\frac{1}{L}$.

\section{Step 1 (D2D):}

For given $\zeta, \quad \lambda_{l}, \quad \mu_{l}$, each D2D link $l$ calculates $p_{D l}^{k^{*}}=\left[\frac{-B_{k l}^{d}+\sqrt{\Delta_{k l}^{d}}}{A_{k l}^{d}}\right]_{0}^{P_{D l}^{\max }}, \forall k \in \mathcal{K}$ and broadcasts the value of $f_{l}^{k}\left(p_{D l}^{k^{*}}\right)$, which is defined in (29).

\section{Step 2 (BS):}

The BS after collecting all values $f_{l}^{k}\left(p_{D l}^{k^{*}}\right), \forall k \in \mathcal{K}, \forall l \in \mathcal{L}$ broadcasts $f_{k}^{\max }=\max _{l \in \mathcal{L}} f_{l}^{k}\left(p_{D l}^{k^{*}}\right)$. to all users.

\section{Step 3 (D2D):}

Each D2D link $l$ performs subchannel allocation by using the following rule

$$
\rho_{l}^{k^{*}}= \begin{cases}1 & \text { if } f_{l}^{k}\left(p_{D l}^{k^{*}}\right) \geq f_{k}^{\max } \\ 0 & \text { otherwise. }\end{cases}
$$

Moreover, it calculates $z_{l}=w_{l} \hat{R}_{D l}\left(\boldsymbol{p}_{D l}, \boldsymbol{\rho}_{l}^{*}\right)-\zeta\left(2 P_{0}^{l}+\right.$ $\left.\alpha_{l} \sum_{k \in \mathcal{N}} \rho_{l}^{k^{*}} p_{D l}^{k^{*}}\right)$, then broadcasts $z_{l}$. By using the received information, each D2D link $l$ updates $\lambda_{l}$ and $\mu_{l}$ according to (38) and (43), and return to Step 1 until convergence.

\section{Step 4 (BS):}

The BS collects information of $z_{l}$, calculates $z_{\min }=\min _{l \in \mathcal{L}} z_{l}$, then broadcasts $z_{\min }$. If $z_{\min }>\zeta$, it updates $\zeta \leftarrow z_{\min }$ and broadcasts $\zeta$. This procedure continues (go back to Step 1) and only terminates if there is no further increase in $\zeta$. The BS then calculates the power allocation for each cellular link as

$$
p_{C k}^{k^{*}}=\frac{\sigma_{k}^{k}+I_{k}}{h_{k k}^{k}}\left(2^{R_{C k}^{\min }}-1\right),
$$

where $I_{k}$ is the estimated interference caused by the co-channel D2D link on channel $k$. Then it broadcasts $p_{C k}^{k^{*}}$ to other cellular links.

The main tasks performed by different network entities can be explained as follows. The BS collects the information regarding $f_{l}^{k}\left(p_{D l}^{k^{*}}\right), \forall k \in \mathcal{K}, \forall l \in \mathcal{L}$ then it broadcasts the maximum value $f_{k}^{\max }=\max _{l \in \mathcal{L}} f_{l}^{k}\left(p_{D l}^{k^{*}}\right)$ for each subchannel (step 2). The BS is also responsible for calculating and broadcasting the updated value of $z_{\min }=\min _{l \in \mathcal{L}} z_{l}$ and $\zeta$ (step 
4). Moreover, as the algorithm converges, the BS calculates the transmit powers of all cellular links and broadcasts the results to the cellular links. Each D2D link $l$ is responsible for calculating the possible power allocation in each subchannel and performing subchannel allocation based on the obtained information. Moreover, it broadcast the values of $f_{l}^{k}\left(p_{D l}^{k^{*}}\right)$ and $z_{l}$ (step 1 and step 3).

\section{Relaxation-BASEd Algorithms}

The dual-based Algorithm 2 has polynomial time complexity; however, it may not achieve the optimal solution. In this section, we propose the optimal $\mathrm{BnB}$ algorithm and the lowcomplexity Relaxation-Based Rounding (RBR) algorithm.

\section{A. Optimal Branch-and-Bound Algorithm}

In this section, we apply the Branch-and-Bound (BnB) approach [38] to develop an algorithm that attains the optimal solution of the original problem. Although the BnB algorithm may not achieve the polynomial time complexity, it can significantly reduce the complexity compared to the exhaustive search algorithm. Since any feasible subchannel allocation variable is binary, we propose the $\mathrm{BnB}$ algorithm by branching the feasible set of the subchannel allocation vectors where each branching iteration is executed by setting an undetermined subchannel allocation variable to a binary value 0 or 1 . Specifically, the algorithm determines the optimal path in the search tree that corresponds to the optimal subbchannel allocations for all D2D links. In addition, this optimal path is decided by iteratively visiting potential nodes in the search tree where each node $m$ is associated with some already determined subchannel allocation variables (corresponding to part of the underlying path connecting node $m$ with the root node) and other undetermined subchannel allocation variables.

Let $\overline{\mathcal{Q}}_{m}$ be the set of all feasible subchannel allocation vectors $\boldsymbol{\rho}$ related to node $m$ where each vector $\boldsymbol{\rho} \in \overline{\mathcal{Q}}_{m}$ contains corresponding determined and undetermined subchannel allocation variables associated with node $m$. For convenience, we use $m$ to indicate the iteration index of the searching procedure, and hence $m=1$ indicates the root node (i.e., we start our search from the root node). Note that each element of $\mathcal{Q}_{1}$ contains all the undetermined subchannel allocation variables.

In each iteration with the corresponding parent node, we consider one of its two child nodes by choosing one undetermined element $\rho_{l}^{k}$ of subchannel assignment vector $\rho$ and set it to a binary value 0 or 1 (called node $m$ ). In node $m$, the local upper-bound, $\mathrm{BU}_{\mathrm{m}}$, and lower-bound, $\mathrm{BL}_{\mathrm{m}}$, must be calculated. We also maintain the global upper-bound, BU*, and lower-bound, $\mathrm{BL}^{*}$, which are, respectively, the highest local upper-bound and local lower-bound of all active nodes in the searching procedure. In a particular node $m$, if the calculated local upper-bound satisfies $\mathrm{BU}_{\mathrm{m}}<\mathrm{BL}^{*}$ then we can remove this node from future consideration because it cannot lead to the optimal solution. On the other hand, if the calculated local lower-bound satisfies $B L_{m}>B L^{*}$, we can update $B L^{*}=B L_{m}$. Furthermore, if the global lower-bound $\mathrm{BL}^{*}$ is sufficiently close to the global upper-bound $\mathrm{BU}^{*}$, we can terminate the algorithm, and output $\mathrm{BL}^{*}$. In the following, we present the procedures to find the local upper-bound and lower-bound in each node $m$ of the algorithm.

1) Upper-bound Calculation: To obtain the upper-bound of node $m$, we take the following procedure. First, we define the set $\mathcal{Q}_{m}$ corresponding to set $\overline{\mathcal{Q}}_{m}$ but any undetermined subchannel allocation variable $\rho_{l}^{k}$ for each element of $\mathcal{Q}_{m}$ is relaxed as $\rho_{l}^{k} \in[0,1]$. Then, we consider the following problem

$$
\begin{aligned}
\max _{\mathbf{p}_{D}, \boldsymbol{\rho} \in \mathcal{Q}_{m}} & \min _{l \in \mathcal{L}} \frac{w_{l} \hat{R}_{D l}\left(\mathbf{p}_{D}, \boldsymbol{\rho}\right)}{P_{D l}^{\text {total }}} \\
\text { s.t. } & (9),(11),(18),
\end{aligned}
$$

whose optimal objective value provides the local upper-bound $\mathrm{BU}_{\mathrm{m}}$ of the resource allocation solution in node $m$. The difference-form of problem (47) can be expressed as

$$
\begin{array}{ll}
\max _{z, \mathbf{p}_{D}, \boldsymbol{\rho} \in \mathcal{Q}_{m}} & z \\
\text { s.t. } & w_{l} \hat{R}_{D l}\left(\mathbf{p}_{D}, \boldsymbol{\rho}\right)-\zeta P_{D l}^{\text {total }}\left(\mathbf{p}_{D}, \boldsymbol{\rho}\right) \geq z, \forall l \in \mathcal{L}, \\
& \text { and }(9),(11),(18) .
\end{array}
$$

We now introduce a new vector $\mathbf{s}_{D}$ that corresponds to the power vector of D2D links $\mathbf{p}_{D}$ and consider the following optimization problem

$$
\begin{array}{ll} 
& \max _{z, \mathbf{s}_{D}, \boldsymbol{\rho} \in \mathcal{Q}_{m}} z \\
\text { s.t. } & w_{l} \bar{R}_{D l}\left(\mathbf{s}_{D l}, \boldsymbol{\rho}\right)-\zeta \bar{P}_{D l}^{\text {total }}\left(\mathbf{s}_{D l}, \boldsymbol{\rho}\right) \geq z, \forall l \in \mathcal{L} \\
& \sum_{k \in \mathcal{K}} s_{D l}^{k} \leq P_{D}^{\max }, \forall l \in \mathcal{L} \\
& \sum_{l \in \mathcal{L}} \rho_{l}^{k} \leq 1, \forall k \in \mathcal{N} \\
& s_{D l}^{k} \leq \rho_{l}^{k} P_{D l k}^{\max }, \forall k \in \mathcal{N}, \forall l \in \mathcal{L}
\end{array}
$$

where

$$
\begin{aligned}
& \bar{R}_{D l}\left(\mathbf{s}_{D l}, \boldsymbol{\rho}\right)=\sum_{k \in \mathcal{N}} \rho_{l}^{k} \log _{2}\left(1+\frac{s_{D l}^{k}}{a_{k l} \rho_{l}^{k}+b_{k l} s_{D l}^{k}}\right) \\
& \bar{P}_{D l}^{\text {total }}=2 P_{0}^{l}+\alpha_{l} \sum_{k \in \mathcal{K}} s_{D l}^{k} .
\end{aligned}
$$

We state one important result in the following proposition.

Proposition 3. Problem (48) and (49) are equivalent, and problem (49) is convex.

Proof. The proof can be found in Appendix D.

Proposition 3 implies that the optimum solution of (48) can be obtained by solving the convex problem (49) using the standard interior point method [34]. We propose Algorithm 3 to solve problem (47), in which we iteratively solve problem (49) for a given $\zeta$ (line 3) and update $\zeta$ (line 4) until convergence. If $\left(\zeta^{*}, \mathbf{s}_{D}^{*}, \boldsymbol{\rho}^{*}\right)$ is the solution obtained from Algorithm 3 then the components of $\mathbf{p}_{D}^{*}$ are given in (73) detailed in Appendix D. We now state another important result in the following proposition.

Proposition 4. The obtained solution $\left(\boldsymbol{p}_{D}^{*}, \boldsymbol{\rho}^{*}\right)$ in Algorithm 3 is the optimal solution of (47).

Proof. See proof in Appendix E. 
Proposition 4 implies that $\zeta$ obtained from Algorithm 3 is an upper-bound of the resource allocation problem associated with node $m$.

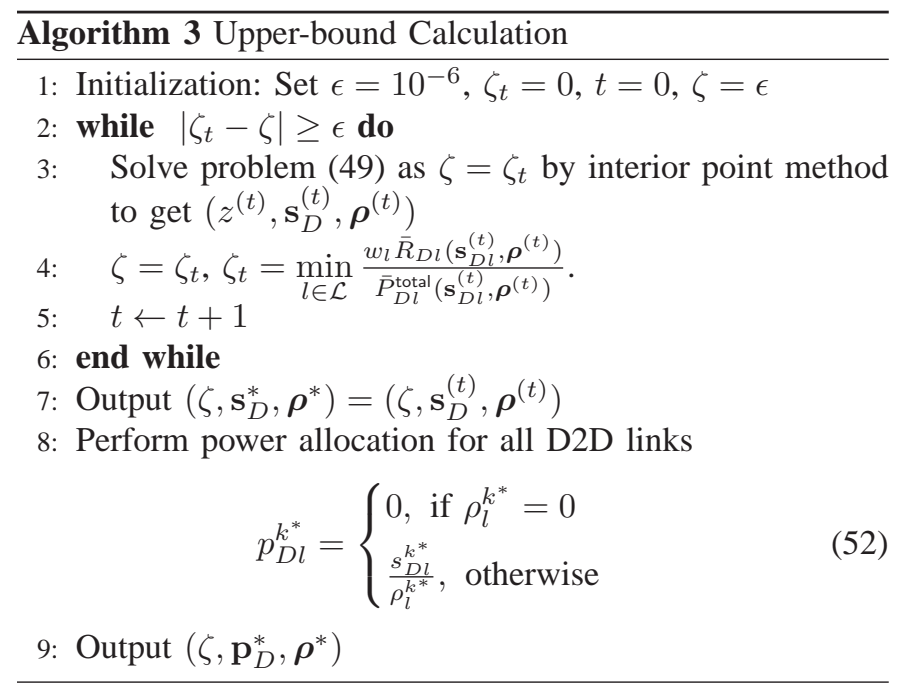

2) Lower-bound Calculation: Note that the local lowerbound in a particular node $m$ can be the objective value achieved by a feasible solution. In node $m$, while determining the local upper-bound, we obtain $\left(\mathbf{s}_{D}^{*}, \boldsymbol{\rho}^{*}\right)$ and $\left(\mathbf{p}_{D}^{*}, \boldsymbol{\rho}^{*}\right)$ in lines 7 and 9 of Algorithm 3, respectively. Since $\rho^{*}$ can contain fractional components, it might not be a feasible solution of problem (47). The local lower-bound in node $m, \mathrm{BL}_{\mathrm{m}}$, can be obtained by rounding off the values of the fractional subchannel allocation variables. The new feasible resource allocation vector $\left(\hat{\mathbf{p}}_{D}, \hat{\boldsymbol{\rho}}\right)$ can be obtained by the following rules

$$
\begin{aligned}
& \hat{\rho}_{l}^{k}=\left\{\begin{array}{l}
1, \text { if } \rho_{l}^{k^{*}}=\max _{l \in \mathcal{L}} \rho_{l}^{k^{*}} \\
0, \text { otherwise, }
\end{array}\right. \\
& \hat{p}_{D l}^{k}=\left\{\begin{array}{l}
0, \text { if } \hat{\rho}_{l}^{k}=0 \\
s_{D l}^{k^{*}}, \text { if } \hat{\rho}_{l}^{k}=1 .
\end{array}\right.
\end{aligned}
$$

Specifically, subchannel $k$ is assigned to D2D link $l$ with highest value of $\rho_{l}^{k^{*}}$. Moreover, the power allocated to subchannel $k$ is equal to $s_{D l}^{k^{*}}$ to ensure the feasibility of the resulting solution. This feasible solution $\left(\hat{\mathbf{p}}_{D}, \hat{\boldsymbol{\rho}}\right)$ is then used to calculate the local lower-bound.

\section{B. Relaxation-Based Rounding Algorithm}

The BnB algorithm may require, in some cases, to visit a large number of nodes. In the following, we propose the Relaxation-Based-Rounding (RBR) algorithm (Algorithm 4), which requires to solve only one relaxed problem and execute the rounding procedure only once. Specifically, we run Algorithm 3 for the root node, which is employed by the $\mathrm{BnB}$ algorithm, to obtain the initial solution in line 1. Based on the obtained result, we perform subchannel allocations for all subchannel $k$ and D2D link $l$ with $\rho_{l}^{k^{*}}=1$ then we execute the rounding procedure (lines 5-10), which is designed to minimize the performance loss as follows. Let $\mathcal{S}_{l}=\left\{k \in \mathcal{N} \mid \rho_{l}^{k^{*}}=1\right\}, \mathcal{S}_{f l}=\left\{k \in \mathcal{N} \mid \rho_{l}^{k^{*}} \in(0,1)\right\}$ be the sets of exclusive and shared subchannels allocated to D2D link $l$, respectively, and $\mathcal{U}_{k}=\left\{l \in \mathcal{L} \mid \rho_{l}^{k^{*}}>0\right\}$ be the set of D2D links with positive subchannel allocation variables on subchannel $k$. In line 2, we calculate the EE of D2D link $l \in \mathcal{L}$ contributed by its exclusive subchannels in set $\mathcal{S}_{l}$ as

$$
\zeta_{l}=\frac{\sum_{k \in \mathcal{S}_{l}} \log _{2}\left(1+\frac{s_{D l}^{k^{*}}}{a_{k l}+s_{D l}^{k^{*}}}\right)}{2 P_{0}+\alpha_{l} \sum_{k \in \mathcal{S}_{l}} s_{D l}^{k^{*}}} .
$$

In lines 8-9, we allocate each shared subchannel to a unique D2D link. First, we calculate the possible EE improvement of each D2D link $l \in \mathcal{L}$ over its shared subchannel $k \in \mathcal{C}_{f}$ as

$$
\begin{aligned}
\Delta_{l}^{k}\left(\mathcal{S}_{l}\right)= & \frac{\sum_{n \in \mathcal{S}_{l} \cup\{k\}} w_{l} \log _{2}\left(1+\frac{s_{l}^{k^{*}}}{a_{k l}+b_{k l} s_{l}^{k^{*}}}\right)}{2 P_{0}+\alpha_{l} \sum_{k \in \mathcal{S}_{l} \cup\{k\}} s_{D l}^{k^{*}}} \\
& -\frac{\sum_{n \in \mathcal{S}_{l}} w_{l} \log _{2}\left(1+\frac{s_{l}^{k^{*}}}{a_{k l}+b_{k l} s_{l}^{k^{*}}}\right)}{2 P_{0}+\alpha_{l} \sum_{k \in \mathcal{S}_{l}} s_{D l}^{k^{*}}},
\end{aligned}
$$

where $\mathcal{S}_{l}$ is the set of subchannels assigned to D2D link $l$ before we consider subchannel $k$ and $\mathcal{C}_{f}$ is the set of unallocated subchannels defined in Algorithm 4.

Now define $\mathcal{S}=\left\{\mathcal{S}_{1}, \cdots, \mathcal{S}_{L}\right\}$ where $\mathcal{S}_{l}$ denotes the set of subchannels allocated to D2D link $l$, and $\mathcal{A}_{k}^{\mathcal{S}}=\{l \in$ $\left.\mathcal{N} \mid \Delta_{l}^{k}\left(\mathcal{S}_{l}\right)>0\right\}$ as the set of D2D links which can improve its $\mathrm{EE}$ if these links are assigned subchannels $k \in \mathcal{C}_{f}$ for a given set $\mathcal{S}$. In the proposed rounding procedure, we sequentially allocate one subchannel $k \in \mathcal{C}_{f}$ to the D2D link in $\mathcal{A}_{k}^{\mathcal{S}}$ that has the minimum EE (line 8). After each assignment, we update the set of assigned subchannels and EE for each D2D link (line 9). The rounding procedure is terminated when all subchannels are allocated.

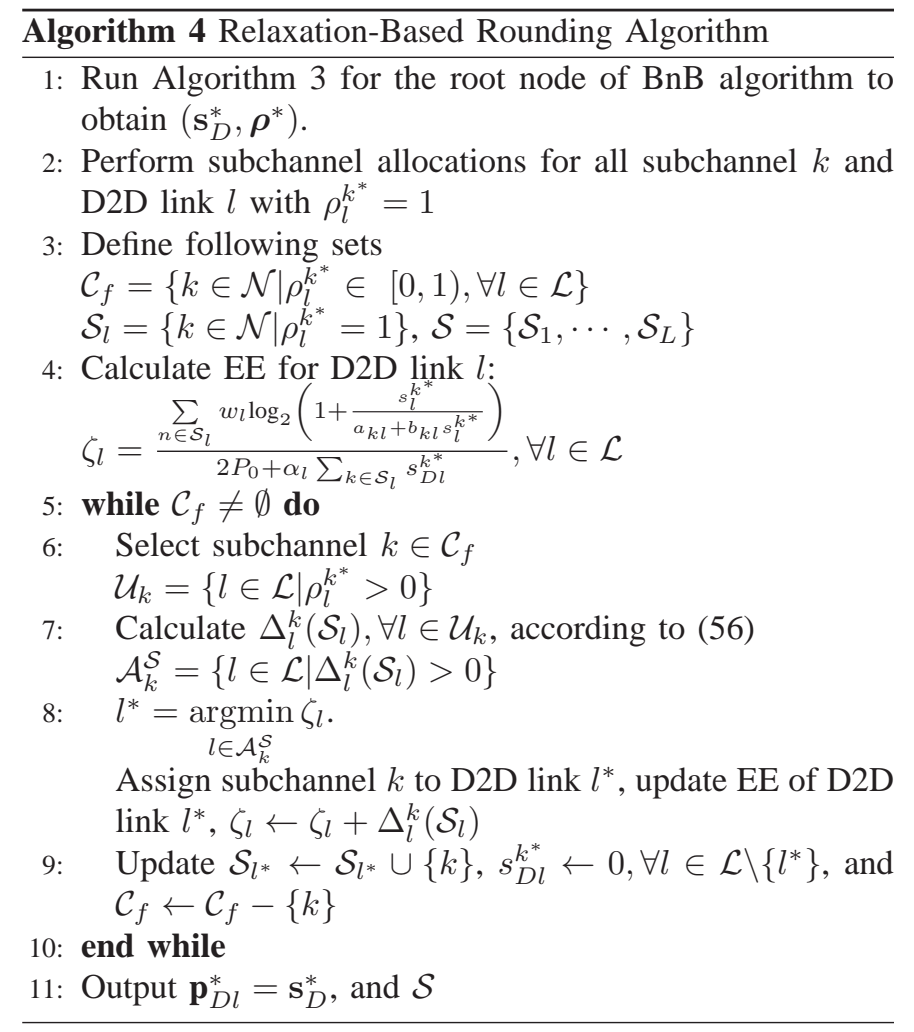


TABLE I

SIMULATION PARAMETERS

\begin{tabular}{|c|c|c|}
\hline Description & Parameter & Value \\
\hline Number of D2D links & $L$ & 2 or 4 \\
Number of cellular links & $K$ & 20 \\
Number of subchannels & $N$ & 20 \\
Maximum distance between Tx and Rx of & $d_{\max }$ & $50 \mathrm{~m}$ \\
D2D links & $P_{C k}^{\max }$ & $0.5 \mathrm{~W}$ \\
Maximum transmit power of cellular link & $P_{D l}^{\max }$ & $0.5 \mathrm{~W}$ \\
Maximum transmit power of D2D link & $P_{0}$ & $0.5 \mathrm{~W}$ \\
Circuit power & $\alpha_{l}$ & 1.5 \\
Scaling factor & $R_{C k}^{\min }$ & $2 \mathrm{~b} / \mathrm{s} / \mathrm{Hz}$ \\
Minimum required rate of cellular links & $w_{l}$ & 1 \\
Weighting parameter & $\sigma_{k}$ & $10^{-12}$ \\
Noise power & & \\
\hline
\end{tabular}

\section{COMPLEXITy ANALYsis}

In this section, we analyze the complexity of the proposed algorithms in term of the number of required arithmetic operations. Since in BnB algorithm, the number of visited nodes is not fixed, its complexity cannot be exactly determined. On the other hand, the dual-based algorithm requires to solve problem (20) iteratively by the dual decomposition method for given $\zeta$ with complexity of $O(N L)$. Number of iterations required to update $\zeta$ has complexity of $O(1)$. Therefore, the complexity of the dual-based algorithm is $O(N L)$.

The Relaxation-Based Rounding algorithm comprises two phases. In the relaxation phase, we iteratively solve problem (49) for given $\zeta$ by the interior-point method with complexity of $O\left(m^{\frac{1}{2}}(m+n) n^{2}\right)$, where $m$ is the number of inequality constraints and $n$ is number of variables [36]. Therefore, the complexity of solving problem (49) and also of the relaxation phase is $O\left(N^{3.5}\right)$. In addition, the rounding phase has complexity of $O\left(L^{2}\right)$. Finally, the complexity of the RBR algorithm is $O\left(N^{3.5}\right)$.

\section{NUMERICAL RESULTS}

We consider the simulation setting shown in Fig. 1 with the base-station located at the center, $K=20$ cellular users, and $L=2$ or 4 D2D links randomly placed in $500 \mathrm{~m} \times 500 \mathrm{~m}$ area, and $N=20$ subchannels for uplink communications. The summary of parameter settings used in the simulations is presented in Table I.

The subchannel power gain is modeled as $h_{k l}^{n}=\left(\frac{d_{k l}}{d_{0}}\right)^{-3} \delta$ where $d_{0}=1 \mathrm{~m}$ is the reference distance, and $d_{k l}>d_{0}$ is the distance between the receiver of link $k$ and the transmitter of link $l$, and $\delta$ represents the Rayleigh fading coefficient, which follows the exponential distribution with the mean value of 1 . We set the noise power equal to $10^{-12} \mathrm{~W}$ for every link. The circuit power of each cellular link $P_{0}$ is $0.5 \mathrm{~W}$, the factor $\alpha_{l}$ is 1.5 for each D2D link, and the maximum transmit powers of each cellular link $k$ and cellular link $l$ are $P_{C k}^{\max }=P_{D l}^{\max }=0.5$ $\mathrm{W}, \forall k \in \mathcal{K}, \forall l \in \mathcal{L}$. In addition, the weighting parameters of D2D links are set as $w_{l}=1, \forall l \in \mathcal{L}$, the maximum distance of D2D links $d_{\max }$ is $50 \mathrm{~m}$, and the minimum required rate of each cellular link $k$ is $R_{C k}^{\min }=R_{C}^{\min }=2 \mathrm{~b} / \mathrm{s} / \mathrm{Hz}, \forall k \in \mathcal{K}$.

We evaluate the performance of the proposed algorithms and that [20] with minor modification (called "conventional"

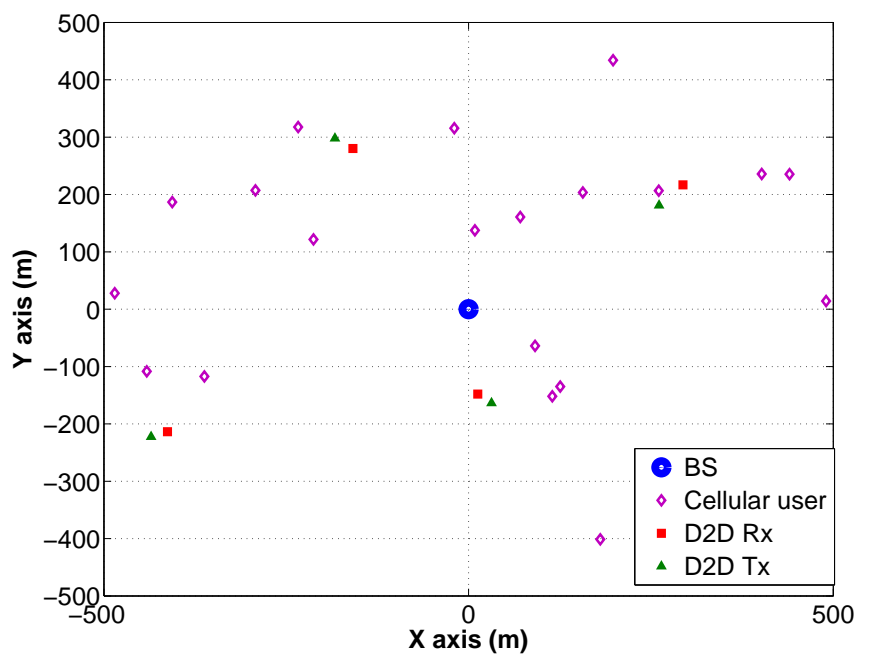

Fig. 1. Simulation setting

algorithm ) since our work and [20] consider the similar network settings, non-orthogonal spectrum sharing between cellular and D2D links, and link EE maximization objective. However, there are some differences between two works. In [20], each D2D or cellular link performs power allocation for all subchannels to maximize the EE while in this work, we consider the joint subchannel assignment and power allocation to maximize the minimum weighted EE of D2D links. Since we focus on maximizing the minimum weighted EE of D2D links while satisfying the minimum cellular link data rates, we modify the algorithm developed in [20] so as to maximize the EE of D2D links, and minimizing the total transmit power of cellular links while maintaining the minimum cellular-link rate requirement.

We also consider the spectrum-efficient solution as a reference, which is obtained by solving problem (48) for the case where all subchannel allocation variables are undetermined and $\zeta=0$. In addition, to verify the efficiency of our algorithms, we compare the objective values achieved by our algorithms with their corresponding upper-bounds achieved by solving the relaxed version of problem (19). All numerical results are acquired by averaging over 1000 random realizations of $\mathrm{D} 2 \mathrm{D}$ and cellular locations, and channel gains. The EE of D2D links corresponding to the $\mathrm{BnB}$ algorithm, dual-based algorithm, RBR algorithm, upper bound as well as the spectrum-efficiency (SE) maximization solution are indicated by "BnB Alg.", "Dual Alg.", "Rounding Alg.", "Upper-bound", and "SE solution", respectively. In all figures in the following, we show the minimum achieved $\mathrm{EE}$ of all D2D links (i.e., the design objective) versus different parameters. For brevity, the minimum EE of all D2D links is simply referred to as EE of D2D links in the figures and following discussions.

Figs. 2 and 3 show the achieved EE of D2D links versus $d_{\max }$ and $R_{C}^{\min }$, respectively, for $L=2$, which allows us to obtain the optimal solution of problem (12) through the $\mathrm{BnB}$ algorithm described in Section V.A within reasonable time. It can be seen that the conventional algorithm and 
SE-maximization solution achieve much lower EE than our proposed energy-efficient algorithms. Moreover, the EE gap between the proposed and conventional algorithms significantly increases as $d_{\max }$ increases. Therefore, it confirms that the proposed algorithms can effectively manage the co-channel interference among the links and make efficient subchannel assignments for D2D links. On the other hand, in the conventional algorithm, since each D2D link selfishly optimizes its $\mathrm{EE}$, the interference among the links may not be well managed. As a result, some D2D links could achieve low EE values, which explains the inferior performance of the conventional design.

It is remarkable that the RBR algorithm performs extremely well with its achieved EE very close to that of the optimal $\mathrm{BnB}$ algorithm. In fact, when the number of D2D links is small, the number of shared subchannels is small as compared to the number of available subchannels, which leads to small performance loss during the rounding phase. Figs. 2 and 3 also indicate that the Dual-Based Algorithm 2 can offer performance close to that of the $\mathrm{BnB}$ algorithm since with a small number of D2D links, the duality gap of problem (22) for given $\zeta$ is also small.

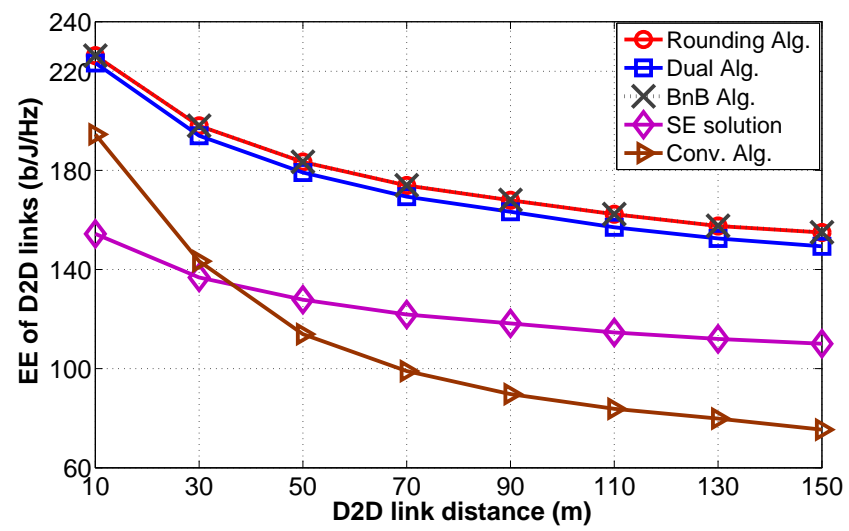

Fig. 2. Minimum EE of D2D links versus $d_{\max }$ for $L=2$

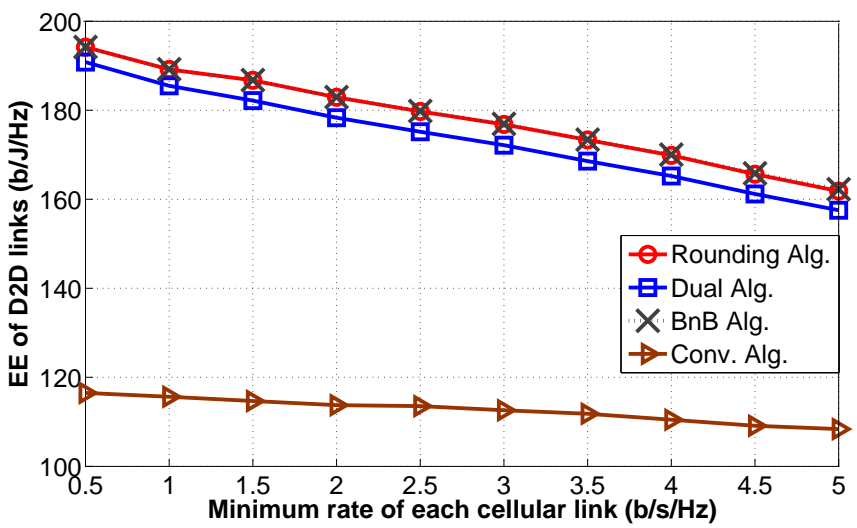

Fig. 3. Minimum EE of D2D links versus minimum rate of cellular links for $L=2$

The characteristics and performance of different algorithms are further investigated with larger number of D2D links,
$L=4$. Figs. 4 and 5 show the convergence of the dualbased Algorithm 2 and the initial relaxation phase of the RBR Algorithm 4 for $d_{\max }=10 \mathrm{~m}$ and $100 \mathrm{~m}$, respectively. They confirm that the gradient-based method used in Algorithm 4 to determine the optimal solution converges faster than the bisection method employed by Algorithm 2.

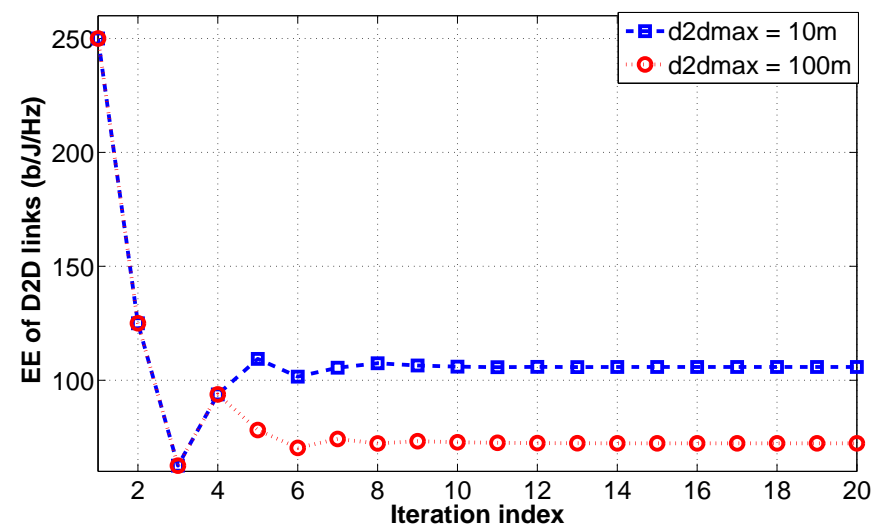

Fig. 4. Convergence behavior of dual-based Algorithm 2

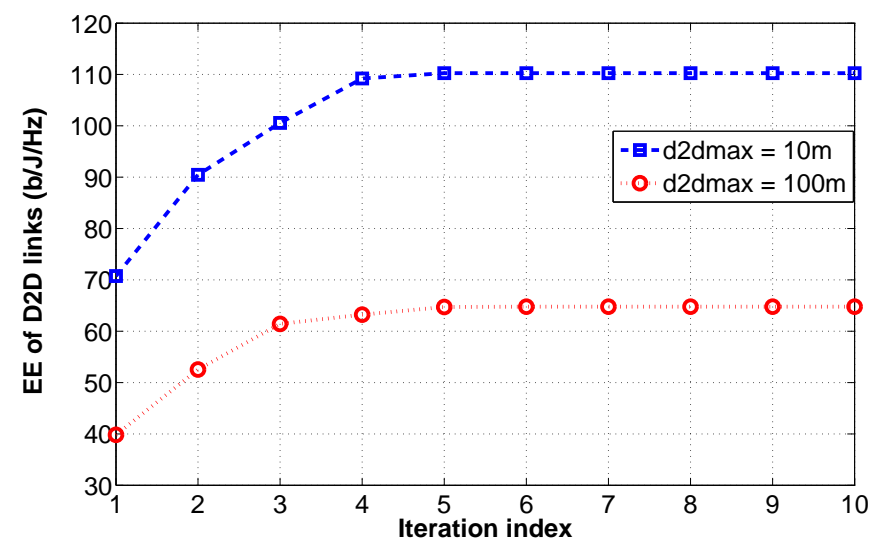

Fig. 5. Convergence behavior of the relaxation phase of RBR Algorithm 4

Fig. 6 indicates that the EE of D2D links achieved by Algorithms 2 and 4 are significantly higher than that of the conventional algorithm, e.g., at $d_{\max }=150 \mathrm{~m}$, Algorithms 2 and 4 can achieve more than $90 \%$ of the upper-bound EE, which is about $300 \%$ that of the conventional algorithm and about $130 \%$ that of the SE-maximization solution.

The achieved EE of D2D links versus the minimum required rate of cellular links, plotted in Fig. 7, indicates that as the required cellular-link rate increases, the achieved EE of D2D links is reduced. This observation can be explained as follows. As the minimum required rate of each cellular link increases, each cellular user has to increase its transmit power to maintain the required rate, which results in stronger interference for the co-channel D2D links. Moreover, since D2D links are relatively robust against interference due to their short communication distances, the minimum EE is moderately impacted as the minimum rate of cellular links increases. 


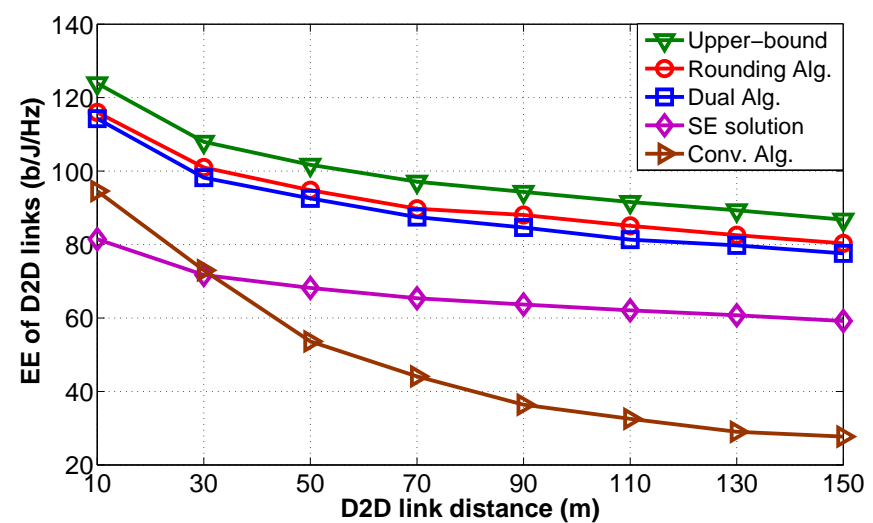

Fig. 6. Minimum EE of D2D links versus D2D link distance when $L=4$

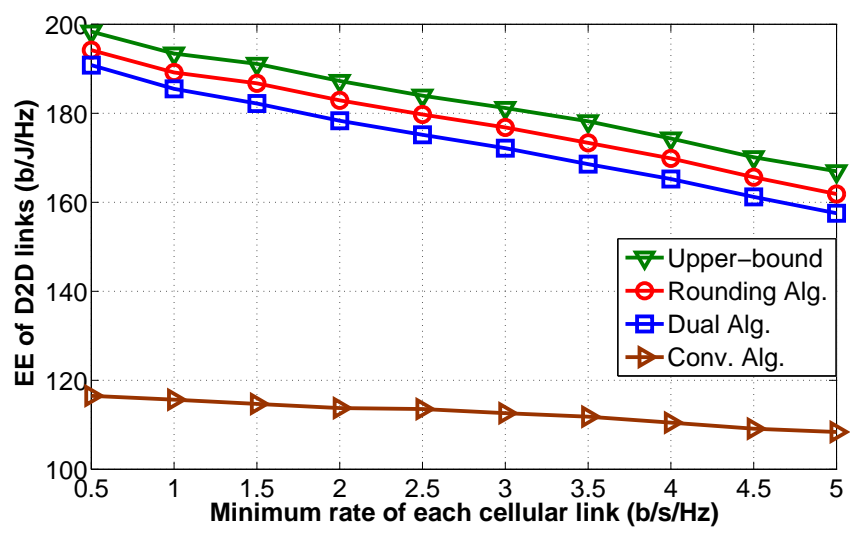

Fig. 7. Minimum EE of D2D links versus minimum required cellular-link rate with $L=4$

Fig. 8 demonstrates the achieved EE of D2D links as a function of the circuit power. Both Algorithm 2 and Algorithm 4 offer excellent performance, which is very close to the upper bound. For small circuit power, their achieved EE is about $150 \%$ of the EE due to the conventional algorithm. However, when the circuit power increases, the performance gap between the proposed and the conventional algorithms is reduced since the total consumed power is dominated by the circuit power.

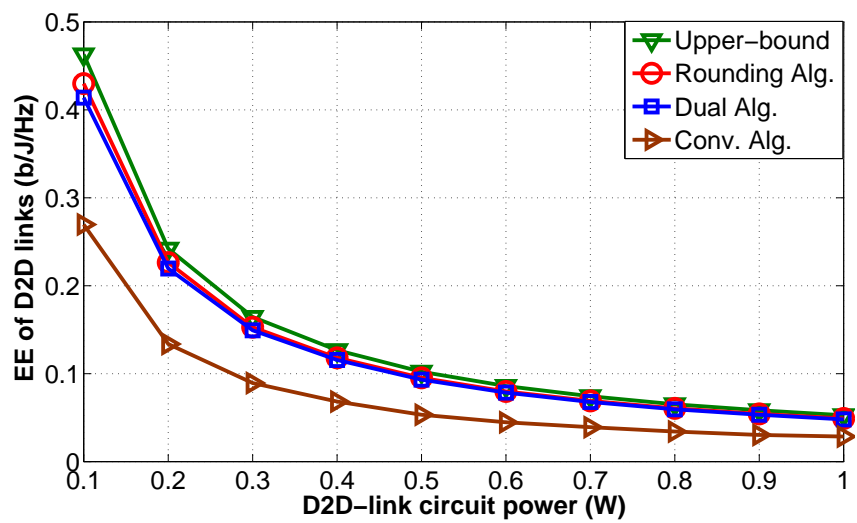

Fig. 8. Minimum EE of D2D links versus circuit power
The achieved EE of D2D links versus the noise power, plotted in Fig. 9 indicates that, for lower noise power, both Algorithms 2 and 4 achieve higher EE, much better than the conventional algorithm. This is because for the proposed algorithms, when $\sigma$ reduces, each D2D or cellular link decreases its transmit power. As a result, the co-channel interference between D2D and cellular links also decreases, which leads to the improvement in the EE. In contrast, in the conventional algorithm, all links would operate in the high-interference regime; therefore, the noise power is more negligible compared to the interference and its variation does not impact the EE achieved by D2D links.

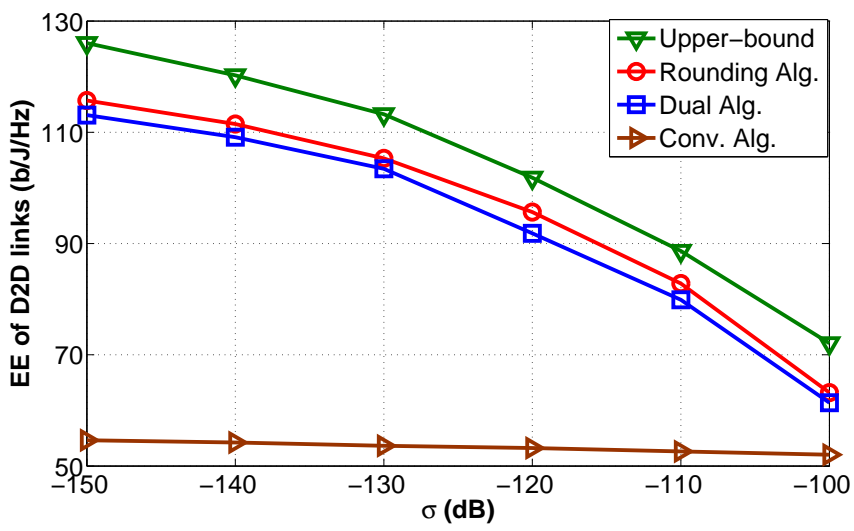

Fig. 9. Minimum EE of D2D links versus the noise power

Finally, Fig. 10 shows that the achieved EE of D2D links decreases as the number of D2D links increases. The performance gap between the proposed and the conventional algorithms also decreases as the number of D2D links increases. This is because as the system supports more D2D links, the available resources for each D2D link becomes smaller, which results in the decrease in the achieved EE of D2D links. Fig. 10 also illustrates that as the number of D2D links increases, the gap between the proposed dual-based algorithm and the upperbound becomes larger since the duality gap of the underlying MINLP at convergence under Algorithm 2 becomes higher, which results in larger performance loss for this algorithm.

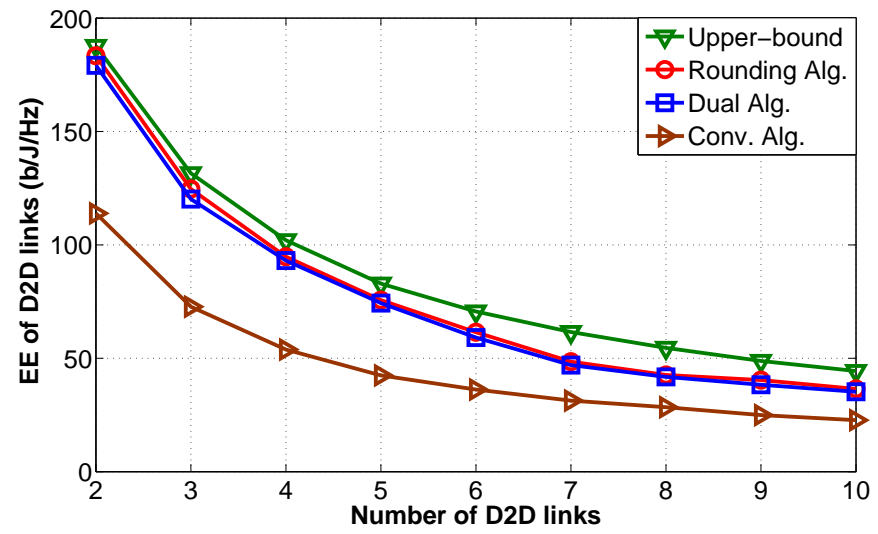

Fig. 10. Minimum EE of D2D links versus number of D2D links 


\section{CONCLUSIONS}

In this paper, we have developed efficient resource allocation algorithms for D2D underlaying cellular systems, which maximizes the minimum weighted $\mathrm{EE}$ of D2D links while guaranteeing the QoS of cellular links. In particular, we have proposed the optimal $\mathrm{BnB}$ algorithm based on the novel branching and bounding procedures, and proposed two low-complexity algorithms: (i) the dual-based Algorithm 2 solves the resource allocation problem in the dual domain, and (ii) the Relaxed-Based Rounding (RBR) Algorithm 4 solves the relaxed version first and then applies a rounding procedure to obtain a feasible solution for the considered resource allocation problem. We have studied the theoretical performance of the proposed low-complexity algorithms and analyzed their computational complexity. Numerical results have confirmed that both proposed Algorithms 2 and 4 can achieve excellent performance, which is close to that due to the optimal $\mathrm{BnB}$ algorithm and the upper bound.

\section{APPENDIX A}

\section{PROOF OF PROPOSITION 1}

We first show that the min-rate constraints of cellular link $k$ must be met at equality as follows:

$$
\log _{2}\left(1+\frac{p_{C k}^{k} h_{k k}^{k}}{\sigma_{k}^{k}+p_{D l}^{k} h_{k l}^{k}}\right)=R_{C k}^{\min }
$$

Note that if $\frac{\sigma_{k}^{k}}{h_{h k}^{k}}\left(2^{R_{C k}^{\min }}-1\right)>P_{C k}^{\max }$, the required minimum rate of cellular link $k$ cannot be supported; hence, problem (12) is infeasible. On the other hand, if $\frac{\sigma_{k}^{k}}{h_{k k}^{k}}\left(2^{R_{C k}^{\min }}-1\right) \leq P_{C k}^{\max }$, the cellular link $k$ can allow D2D link $l$ to reuse its resource. Therefore, the power of cellular link $k$ can be expressed as the power of D2D link $l$ on subchannel $k$ as follows:

$$
\begin{aligned}
& p_{C k}^{k}=\frac{\sigma_{k}^{k}+p_{D l}^{k} h_{k l}^{k}}{h_{k k}^{k}}\left(2^{R_{C k}^{\min }}-1\right) \\
& p_{D l}^{k}=\frac{1}{h_{k l}^{k}}\left(\frac{p_{C k}^{k} h_{k k}^{k}}{2^{R_{C k}^{\mathrm{min}}}-1}-\sigma_{k}^{k}\right)
\end{aligned}
$$

Moreover, the power of cellular link $k$ must satisfy the maximum power constraint, which can be expressed as

$$
p_{D l}^{k} \leq \frac{1}{h_{k l}^{k}}\left(\frac{P_{C k}^{\max } h_{k k}^{k}}{2^{R_{C k}^{\min }}-1}-\sigma_{k}^{k}\right) .
$$

Let us now define

$$
P_{D l k}^{\max } \triangleq \min \left\{P_{D l}^{\max }, \frac{1}{h_{k l}^{k}}\left(\frac{P_{C k}^{\max } h_{k k}^{k}}{2^{R_{C k}^{\min }}-1}-\sigma_{k}^{k}\right)\right\}
$$

Then, it can be verified that if D2D link $l \in \mathcal{L}$ reuses the resource of cellular link $k$, we have $p_{D l}^{k} \in\left[0, P_{D l k}^{\max }\right]$, and $p_{D l}^{k}=\frac{1}{h_{k l}^{k}}\left(\frac{p_{C k}^{k} h_{k k}^{k}}{2^{R_{C k}^{m i n}-1}}-\sigma_{k}^{k}\right)$.

\section{APPENDIX B \\ ProOf OF THEOREM 1}

First, we prove that $\eta^{*}(\zeta)$ is a decreasing function of $\zeta$. Suppose we have $\zeta_{1}>\zeta_{2}$, and $\eta^{*}\left(\zeta_{1}\right)$ and $\eta^{*}\left(\zeta_{2}\right)$ are the optimal objective value of problem (20) corresponding to the parameters $\zeta_{1}$ and $\zeta_{2}$, respectively. Now, we define the following

$$
\left(\mathbf{p}_{D}^{o}, \boldsymbol{\rho}^{o}\right) \triangleq \underset{\left(\mathbf{p}_{D}, \boldsymbol{\rho}\right) \in \mathcal{D}}{\operatorname{argmax}} \min _{l \in \mathcal{L}}\left[w_{l} \hat{R}_{D l}\left(\mathbf{p}_{D}, \boldsymbol{\rho}\right)-\zeta_{1} P_{D l}^{\text {total }}\left(\mathbf{p}_{D}, \boldsymbol{\rho}\right)\right] .
$$

Then, we have

$$
w_{l} \hat{R}_{D l}\left(\mathbf{p}_{D}^{o}, \boldsymbol{\rho}^{o}\right)-\zeta_{1} P_{D l}^{\text {total }}\left(\mathbf{p}_{D}^{o}, \boldsymbol{\rho}^{o}\right) \geq \eta^{*}\left(\zeta_{1}\right), \forall l \in \mathcal{L} .
$$

Because $P_{D l}^{\text {total }}\left(\mathbf{p}_{D}^{o}, \boldsymbol{\rho}^{o}\right) \geq 0$ and $\zeta_{1}>\zeta_{2}$, we have $\zeta_{1} P_{D l}^{\text {total }}\left(\mathbf{p}_{D}^{o}, \boldsymbol{\rho}^{o}\right) \geq \zeta_{2} P_{D l}^{\text {total }}\left(\mathbf{p}_{D}^{o}, \boldsymbol{\rho}^{o}\right), \forall l \in \mathcal{L}$. Consequently, we have $w_{l} \hat{R}_{D l}\left(\mathbf{p}_{D}^{o}, \boldsymbol{\rho}^{o}\right)-\zeta_{1} P_{D l}^{\text {total }}\left(\mathbf{p}_{D}^{o}, \boldsymbol{\rho}^{o}\right) \leq$ $w_{l} \hat{R}_{D l}\left(\mathbf{p}_{D}^{o}, \boldsymbol{\rho}^{o}\right)-\zeta_{2} P_{D l}^{\text {total }}\left(\mathbf{p}_{D}^{o}, \boldsymbol{\rho}^{o}\right), \forall l \in \mathcal{L}$. Finally, we arrive at the following

$$
\begin{aligned}
\eta^{*}\left(\zeta_{2}\right) & =\max _{\left(\mathbf{p}_{D}, \boldsymbol{\rho}\right) \in \mathcal{D}} \min _{l \in \mathcal{L}}\left[w_{l} \hat{R}_{D l}\left(\mathbf{p}_{D}, \boldsymbol{\rho}\right)-\zeta_{2} P_{D l}^{\text {total }}\left(\mathbf{p}_{D}, \boldsymbol{\rho}\right)\right] \\
& \geq \min _{l \in \mathcal{L}}\left[w_{l} \hat{R}_{D l}\left(\mathbf{p}_{D}^{o}, \boldsymbol{\rho}^{o}\right)-\zeta_{2} P_{D l}^{\text {total }}\left(\mathbf{p}_{D}^{o}, \boldsymbol{\rho}^{o}\right)\right] \\
& \geq \min _{l \in \mathcal{L}}\left[w_{l} \hat{R}_{D l}\left(\mathbf{p}_{D}^{o}, \boldsymbol{\rho}^{o}\right)-\zeta_{1} P_{D l}^{\text {total }}\left(\mathbf{p}_{D}^{o}, \boldsymbol{\rho}^{o}\right)\right] \\
& =\eta^{*}\left(\zeta_{1}\right)
\end{aligned}
$$

From these results, we have $\eta^{*}\left(\zeta_{2}\right) \geq \eta^{*}\left(\zeta_{1}\right), \forall \zeta_{1}>\zeta_{2}$, which implies that $\eta^{*}(\zeta)$ is a decreasing function of $\zeta$.

Assume that (21) holds, we need to prove that $\zeta^{*}$ is the optimal solution of problem (19). We prove this by contradiction as follows. If $\zeta^{*}$ is not the optimal solution of problem (19), then we have $\exists \zeta^{o}=\min _{l \in \mathcal{L}} \frac{w_{l} \hat{R}_{D l}\left(\mathbf{p}_{D}^{o}, \boldsymbol{\rho}^{o}\right)}{P_{D l}^{\text {total }}\left(\mathbf{p}_{D}^{o}, \boldsymbol{\rho}^{o}\right)}$, where $\zeta^{o}>\zeta^{*}$ be the optimal solution of problem (19). This means that $\frac{w_{l} \hat{R}_{D l}\left(\mathbf{p}_{D}^{o}, \rho^{o}\right)}{P_{D l}^{\text {total }}\left(\mathbf{p}_{D}^{o}, \boldsymbol{\rho}^{o}\right)}>\zeta^{*}, \forall l \in \mathcal{L}$, which implies that $w_{l} \hat{R}_{D l}\left(\mathbf{p}_{D}^{o}, \boldsymbol{\rho}^{o}\right)-\zeta^{*} P_{D l}^{\text {total }}\left(\mathbf{p}_{D}^{o}, \boldsymbol{\rho}^{o}\right)>0, \forall l \in \mathcal{L}$. Therefore, we have

$$
\begin{aligned}
& \max _{\mathbf{p}_{D}, \boldsymbol{\rho} \in \mathcal{D}} \min _{l \in \mathcal{L}}\left[w_{l} \hat{R}_{D l}\left(\mathbf{p}_{D}, \boldsymbol{\rho}\right)-\zeta^{*} P_{D l}^{\text {total }}\left(\mathbf{p}_{D}, \boldsymbol{\rho}\right)\right] \\
& \quad \geq \min _{l \in \mathcal{L}}\left[w_{l} \hat{R}_{D l}\left(\mathbf{p}_{D}, \boldsymbol{\rho}\right)-\zeta^{*} P_{D l}^{\text {total }}\left(\mathbf{p}_{D}, \boldsymbol{\rho}\right)\right] \\
& \quad>0,
\end{aligned}
$$

which contradicts with the assumption in (21). Therefore, $\zeta^{*}=$ $\min _{l \in \mathcal{L}} \frac{w_{l} \hat{R}_{D l}\left(\mathbf{p}_{D}^{*}, \boldsymbol{\rho}^{*}\right)}{P_{D l}^{\text {totat }}\left(\mathbf{p}_{D}^{*}, \boldsymbol{\rho}^{*}\right)}$ is the optimal solution of problem (12).

\section{APPENDIX C PROOF OF PROPOSITION 2}

Since the dual decomposition algorithm proposed to solve problem (20) for given $\zeta$ always converges if we choose the step sizes $\boldsymbol{\theta}$ and $\boldsymbol{\kappa}$ appropriately [35], and the bisection method to update $\zeta$ always converges, the iterative loops of Algorithm 2 always converge. Hence, Algorithm 2 returns a feasible solution of problem (19).

From the dual decomposition procedure, we have

$$
\begin{aligned}
\left(\mathbf{p}_{D}^{*}, \boldsymbol{\rho}^{*}\right)=\underset{\mathbf{p}_{D} \in \mathcal{X}, \boldsymbol{\rho} \in \mathcal{C}}{\operatorname{argmax}} & \sum_{l \in \mathcal{L}} \mu_{l}^{*}\left[w_{l} \hat{R}_{D l}\left(\mathbf{p}_{D}, \boldsymbol{\rho}\right)-\zeta^{*} P_{D l}^{\text {total }}\right] \\
& +\sum_{l \in \mathcal{L}} \lambda_{l}^{*}\left(P_{D}^{\max }-\sum_{k \in \mathcal{N}} \rho_{l}^{k} p_{D l}^{k}\right) .
\end{aligned}
$$


We denote $\mathcal{X}_{f}=\left\{\mathbf{p}_{D} \in \mathcal{X} \mid \sum_{k \in \mathcal{N}} \rho_{l}^{k} p_{D l}^{k} \leq P_{D l}^{\max }, \boldsymbol{\rho} \in \mathcal{C}\right\}$ as the set of feasible power allocation solutions of problem (19); hence, $\mathcal{X}_{f} \subset \mathcal{X}$. Then, we have

$$
\begin{aligned}
& \max _{\mathbf{p}_{D} \in \mathcal{X}_{f}, \boldsymbol{\rho} \in \mathcal{C}} \sum_{l \in \mathcal{L}} \mu_{l}^{*}\left[w_{l} \hat{R}_{D l}\left(\mathbf{p}_{D}, \boldsymbol{\rho}\right)-\zeta^{*} P_{D l}^{\text {total }}\left(\mathbf{p}_{D}, \boldsymbol{\rho}\right)\right] \\
& \stackrel{(\text { a) }}{\leq} \max _{\mathbf{p}_{D} \in \mathcal{X}_{f}, \boldsymbol{\rho} \in \mathcal{C}} \sum_{l \in \mathcal{L}} \mu_{l}^{*}\left[w_{l} \hat{R}_{D l}\left(\mathbf{p}_{D}, \boldsymbol{\rho}\right)-\zeta^{*} P_{D l}^{\text {total }}\left(\mathbf{p}_{D}, \boldsymbol{\rho}\right)\right] \\
&+\sum_{l \in \mathcal{L}} \lambda_{l}^{*}\left(P_{D}^{\max }-\sum_{k \in \mathcal{N}} \rho_{l}^{k} p_{D l}^{k}\right) \\
& \stackrel{\text { (b) } \max _{\mathbf{p}_{D} \in \mathcal{X}, \boldsymbol{\rho} \in \mathcal{C}}}{ } \sum_{l \in \mathcal{L}} \mu_{l}^{*}\left[w_{l} \hat{R}_{D l}\left(\mathbf{p}_{D}, \boldsymbol{\rho}\right)-\zeta^{*} P_{D l}^{\text {total }}\left(\mathbf{p}_{D}, \boldsymbol{\rho}\right)\right] \\
&+\sum_{l \in \mathcal{L}} \lambda_{l}^{*}\left(P_{D}^{\max }-\sum_{k \in \mathcal{N}} \rho_{l}^{k} p_{D l}^{k}\right)
\end{aligned}
$$

$\stackrel{(\mathrm{c})}{=} 0$.

Inequality $(a)$ holds because of $\sum_{l \in \mathcal{L}} \lambda_{l}^{*}\left(P_{D}^{\max }-\sum_{k \in \mathcal{N}} \rho_{l}^{k} p_{D l}^{k}\right) \geq$ 0 ; inequality $(b)$ is the result of $\mathcal{X}_{f} \subset \mathcal{X}$; and equality $(c)$ is due to the assumption of Proposition 2. Therefore, we have

$\max _{\mathbf{p}_{D} \in \mathcal{X}_{f}, \boldsymbol{\rho} \in \mathcal{C}} \sum_{l \in \mathcal{L}} \mu_{l}^{*}\left[w_{l} \hat{R}_{D l}\left(\mathbf{p}_{D}, \boldsymbol{\rho}\right)-\zeta^{*} P_{D l}^{\text {total }}\left(\mathbf{p}_{D}, \boldsymbol{\rho}\right)\right]=0$.

Since we have $\mu_{l}^{*}>0, \forall l \in \mathcal{L}$, the following holds

$$
\max _{\mathbf{p}_{D} \in \mathcal{X}_{f}, \boldsymbol{\rho} \in \mathcal{C}} \min _{l \in \mathcal{L}}\left[w_{l} R_{D l}\left(\mathbf{p}_{D}, \boldsymbol{\rho}\right)-\zeta^{*} P_{D l}^{\text {total }}\left(\mathbf{p}_{D}, \boldsymbol{\rho}\right)\right]=0 .
$$

Therefore, by using the results of Theorem $1, \zeta^{*}$ is the optimal solution of problem (19).

\section{APPENDIX D}

\section{ProOF OF PROPOSITION 3}

If $\left(z^{*}, \mathbf{p}_{D}^{*}, \boldsymbol{\rho}^{*}\right)$ is the optimal solution of problem (48), we can express $\mathbf{s}_{D}^{*}$ as $s_{D l}^{k^{*}}=\rho_{l}^{k^{*}} p_{D l}^{k^{*}}, \forall l \in \mathcal{L}, \forall k \in \mathcal{N}$. Therefore, $\left(z^{*}, \mathbf{s}_{D}^{*}, \boldsymbol{\rho}^{*}\right)$ is a feasible solution of problem (49). On the other hand, if $\left(z^{*}, \mathbf{s}_{D}^{*}, \boldsymbol{\rho}^{*}\right)$ is the optimal solution of problem (49), $\mathbf{p}_{D}^{*}$ is given as

$$
p_{D l}^{k^{*}}=\left\{\begin{array}{l}
0, \text { if } \rho_{l}^{k^{*}}=0 \\
\frac{s_{D l}^{k^{*}}}{\rho_{l}^{k^{*}}}, \text { otherwise. }
\end{array}\right.
$$

Consequently, $\left(z^{*}, \mathbf{p}_{D}^{*}, \boldsymbol{\rho}^{*}\right)$ is a feasible solution of problem (48). Hence, $z^{*}$ is the optimal objective value of problem (48) iff it is the optimal objective value of problem (49), which means that problems (48) and (49) are equivalent.

In the following, we prove that (48) is convex. It can be seen that in problem (48), the objective is a linear function of variable $z$, and $(49 \mathrm{c})-(49 \mathrm{e})$ are the linear constraints. Therefore, we only need to prove that $g_{l}\left(\mathbf{s}_{D l}, \boldsymbol{\rho}\right)$ are concave functions of $\left(\mathbf{s}_{D l}, \boldsymbol{\rho}\right), \forall l \in \mathcal{L}$ where

$$
g_{l}\left(\mathbf{s}_{D l}, \boldsymbol{\rho}\right) \triangleq w_{l} \bar{R}_{D l}\left(\mathbf{s}_{D l}, \boldsymbol{\rho}\right)-\zeta \bar{P}_{D l}^{\text {total }}\left(\mathbf{s}_{D l}, \boldsymbol{\rho}\right) .
$$

Moreover, in $g_{l}\left(\mathbf{s}_{D l}, \boldsymbol{\rho}\right), \bar{P}_{D l}^{\text {total }}\left(\mathbf{s}_{D l}, \boldsymbol{\rho}\right)=2 P_{0}^{l}+\alpha_{l} \sum_{k \in \mathcal{K}} s_{D l}^{k}$ is a linear combination of $s_{D l}$. Therefore, the remaining task is to prove that $\bar{R}_{D l}\left(\mathbf{s}_{D l}, \boldsymbol{\rho}\right)$ are concave functions of $\left(\mathbf{s}_{D l}, \boldsymbol{\rho}\right), \forall l \in$ $\mathcal{L}$.
We also have $\bar{R}_{D l}\left(\mathbf{s}_{D l}, \boldsymbol{\rho}\right)=\sum_{k \in \mathcal{K}} \bar{R}_{D l}^{k}\left(s_{D l}^{k}, \rho_{l}^{k}\right)$ where

$$
\bar{R}_{D l}^{k}\left(s_{D l}^{k}, \rho_{l}^{k}\right)=\left\{\begin{array}{l}
\rho_{l}^{k} \log _{2}\left(1+\frac{s_{D l}^{k}}{a_{k l} \rho_{l}^{k}+b_{k l} s_{D l}^{k}}\right), \\
\quad \text { if } \rho_{l}^{k} \text { undetermined } \\
\log _{2}\left(1+\frac{s_{D l}^{k}}{a_{k l}+b_{k l} s_{D l}^{k}}\right), \text { if } \rho_{l}^{k}=1 \\
0, \text { if } \rho_{l}^{k}=0 .
\end{array}\right.
$$

In the following, we will prove that $\bar{R}_{D l}^{k}\left(s_{D l}^{k}, \rho_{l}^{k}\right)$ are concave functions for all possible cases of $\rho_{l}^{k}$. First, if $\rho_{l}^{k}=1$, then $\bar{R}_{D l}^{k}\left(s_{D l}^{k}, \rho_{l}^{k}\right)=\log _{2}\left(1+\frac{s_{D l}^{k}}{a_{k l}+b_{k l} s_{D l}^{k}}\right)$ and the second partial derivative of $\bar{R}_{D l}^{k}\left(s_{D l}^{k}, \rho_{l}^{k}\right)$ can be expressed as

$$
\begin{aligned}
& \frac{\partial^{2} \bar{R}_{D l}^{k}\left(s_{D l}^{k}, \rho_{l}^{k}\right)}{\partial s_{D l}^{{ }^{2}}}= \\
& -\frac{a_{k l} b_{k l}\left[a_{k l}+\left(b_{k l}+1\right) s_{D l}^{k}\right]+a_{k l}\left(b_{k l}+1\right)\left(a_{k l}+b_{k l} s_{D l}^{k}\right)}{\left(a_{k l}+b_{k l} s_{D l}^{k}\right)^{2}\left[a_{k l}+\left(b_{k l}+1\right) s_{D l}^{k}\right]^{2}}
\end{aligned}
$$

Since $a_{k l}>0$ and $b_{k l} \geq 0, \forall k \in \mathcal{K}, l \in \mathcal{L}$, we have $\frac{\partial^{2} \bar{R}_{D l}^{k}\left(s_{D l}^{k}, \rho_{l}^{k}\right)}{\partial s_{D L}^{2}} \leq 0$ for all non-negative values of $s_{D l}^{k}$. As a result, $R_{D l}^{k}\left(s_{D l}^{k}, \rho_{l}^{k}\right)$ is a concave function of $s_{D l}^{k}$ for given $\rho_{l}^{k}=1$.

We now consider the case where $\rho_{l}^{k}$ is an undetermined variable. Since $\bar{g}_{l}^{k}\left(s_{D l}^{k}\right)=\log _{2}\left(1+\frac{s_{D l}^{k}}{a_{k l}+b_{k l} s_{D l}^{k}}\right)$ is a concave function of variable $s_{D l}^{k}$, the related function $g_{l}^{k}\left(s_{D l}^{k}, \rho_{l}^{k}\right)=$ $\rho_{l}^{k} \bar{g}_{l}^{k}\left(\frac{s_{D l}^{k}}{\rho_{l}^{k}}\right)=\rho_{l}^{k} \log _{2}\left(1+\frac{s_{D l}^{k}}{a_{k l} \rho_{l}^{k}+b_{k l} s_{D l}^{k}}\right)$ is also a concave function of $\left(s_{D l}^{k}, \rho_{l}^{k}\right), \forall \rho_{l}^{k}>0$. In addition, for the given $s_{D l}^{k}$

$$
\begin{aligned}
& \lim _{\rho_{l}^{k} \rightarrow 0^{+}} \rho_{l}^{k} \log _{2}\left(1+\frac{s_{D l}^{k}}{a_{k l} \rho_{l}^{k}+b_{k l} s_{D l}^{k}}\right) \\
& =\lim _{\rho_{l}^{k} \rightarrow 0^{+}} \rho_{l}^{k} \log _{2}\left(1+\frac{1}{a_{k l} \frac{\rho_{l}^{k}}{s_{D l}^{k}}+b_{k l}}\right)=0 .
\end{aligned}
$$

Hence, $g_{l}^{k}\left(s_{D l}^{k}, \rho_{l}^{k}\right)$ is a continuous function of $\rho_{l}^{k}$. According to [37], the concavity of $g_{l}^{k}\left(s_{D l}^{k}, \rho_{l}^{k}\right)$ is preserved in the boundary of its domain. Therefore, $g_{l}^{k}\left(s_{D l}^{k}, \rho_{l}^{k}\right)$ is a concave function of $\left(s_{D l}^{k}, \rho_{l}^{k}\right)$. As a result, $\bar{R}_{D l}^{k}\left(s_{D l}^{k}, \rho_{l}^{k}\right)$ is a concave function of $\left(s_{D l}^{k}, \rho_{l}^{k}\right)$. Finally, since $\bar{R}_{D l}^{k}\left(s_{D l}^{k}, \rho_{l}^{k}\right)$ is a concave function for all the cases of $\rho_{l}^{k}, g_{l}\left(\mathbf{s}_{D l}, \boldsymbol{\rho}\right)$ is a concave function for all $l \in \mathcal{L}$, which means that problem (48) is a convex optimization problem.

\section{APPENDIX E PROOF OF PROPOSITION 4}

Because problems (48) and (49) are equivalent for given node $m$ and $\mathcal{Q}_{m}$, from the solution of problem (49) we can obtain the solution of problem (48). Assume that $\left(z^{(t-1)}, \mathbf{p}_{D}^{(t-1)}, \boldsymbol{\rho}^{(t-1)}\right)$ and $\left(z^{(t)}, \mathbf{p}_{D}^{(t)}, \boldsymbol{\rho}^{(t)}\right)$ are the solution of problem (48) in iterations $t-1$ and $t$ respectively. In 
addition, let us define $\zeta_{t}=\min _{l \in \mathcal{L}} \frac{w_{l} \hat{R}_{D l}\left(\mathbf{p}_{D}^{(t)}, \boldsymbol{\rho}^{(t)}\right)}{P_{D l}^{\text {(total }}\left(\mathbf{p}_{D}^{(t)}, \boldsymbol{\rho}^{(t)}\right)}$, and $\zeta_{t-1}=$ $\min _{l \in \mathcal{L}} \frac{w_{l} \hat{R}_{D l}\left(\mathbf{p}_{D}^{(t-1)}, \boldsymbol{\rho}^{(t-1)}\right)}{P_{D l}^{\text {(total }}\left(\mathbf{p}_{D}^{(t-1)}, \boldsymbol{\rho}^{(t-1)}\right)}$. Moreover, we have

$$
\begin{aligned}
& \max _{\left(\mathbf{p}_{D}, \boldsymbol{\rho}\right) \in \mathcal{D}} \min _{l \in \mathcal{L}} w_{l} \hat{R}_{D l}\left(\mathbf{p}_{D}, \boldsymbol{\rho}\right)-\zeta_{t-1} P_{D l}^{\text {total }}\left(\mathbf{p}_{D}, \boldsymbol{\rho}\right) \\
= & \min _{l \in \mathcal{L}} w_{l} \hat{R}_{D l}\left(\mathbf{p}_{D}^{(t)}, \boldsymbol{\rho}^{(t)}\right)-\zeta_{t} P_{D l}^{\text {total }}\left(\mathbf{p}_{D}^{(t)}, \boldsymbol{\rho}^{(t)}\right) \\
\geq & \min _{l \in \mathcal{L}} w_{l} \hat{R}_{D l}\left(\mathbf{p}_{D}^{(t-1)}, \boldsymbol{\rho}^{(t-1)}\right)-\zeta_{t-1} P_{D l}^{\text {total }}\left(\mathbf{p}_{D}^{(t-1)}, \boldsymbol{\rho}^{(t-1)}\right) \\
= & 0
\end{aligned}
$$

where $\mathcal{D}$ is the set of feasible solutions of problem (48). Therefore, $\min _{l \in \mathcal{L}} w_{l} \hat{R}_{D l}\left(\mathbf{p}_{D}^{(t)}, \boldsymbol{\rho}^{(t)}\right)-\zeta_{t} P_{D l}^{\text {total }}\left(\mathbf{p}_{D}^{(t)}, \boldsymbol{\rho}^{(t)}\right) \geq 0$, which means that $\zeta_{t}=\min _{l \in \mathcal{L}} \frac{w_{l} \hat{R}_{D l}\left(\mathbf{p}_{D}^{(t)}, \boldsymbol{\rho}^{(t)}\right)}{P_{D l}^{\text {total }}\left(\mathbf{p}_{D}^{(t)}, \boldsymbol{\rho}^{(t)}\right)} \geq \zeta_{t-1}$. This implies that Algorithm 3 creates a sequence of feasible solutions of problem (48) whose objective values monotonically increase over iterations; therefore, the algorithm converges. Assume that at convergence, $\zeta_{t-1}=\zeta_{t}=\zeta^{*}=\min _{l \in \mathcal{L}} \frac{w_{l} \hat{R}_{D l}\left(\mathbf{p}_{D}^{*}, \boldsymbol{\rho}^{*}\right)}{P_{D l}^{\text {total }}\left(\mathbf{p}_{D}^{*}, \boldsymbol{\rho}^{*}\right)}$. Therefore, the following must hold

$$
\begin{aligned}
& \max _{\left(\mathbf{p}_{D}, \boldsymbol{\rho}\right) \in \mathcal{D}} \min _{l \in \mathcal{L}}\left[w_{l} \hat{R}_{D l}\left(\mathbf{p}_{D}, \boldsymbol{\rho}\right)-\zeta_{t-1} P_{D l}^{\text {total }}\left(\mathbf{p}_{D}, \boldsymbol{\rho}\right)\right] \\
= & \min _{l \in \mathcal{L}}\left[w_{l} \hat{R}_{D l}\left(\mathbf{p}_{D}^{(t)}, \boldsymbol{\rho}^{(t)}\right)-\zeta_{t-1} P_{D l}^{\text {total }}\left(\mathbf{p}^{(t)}, \boldsymbol{\rho}^{(t)}\right)\right] \\
= & \min _{l \in \mathcal{L}}\left[w_{l} \hat{R}_{D l}\left(\mathbf{p}_{D}^{(t)}, \boldsymbol{\rho}^{(t)}\right)-\zeta_{t} P_{D l}^{\text {total }}\left(\mathbf{p}^{(t)}, \boldsymbol{\rho}^{(t)}\right)\right] \\
= & \min _{l \in \mathcal{L}}\left[w_{l} \hat{R}_{D l}\left(\mathbf{p}_{D}^{*}, \boldsymbol{\rho}^{*}\right)-\zeta^{*} P_{D l}^{\text {total }}\left(\mathbf{p}^{*}, \boldsymbol{\rho}^{*}\right)\right] \\
= & 0 .
\end{aligned}
$$

Since $\left(\zeta^{*}, \mathbf{p}_{D}^{*}, \boldsymbol{\rho}^{*}\right)$ satisfies the sufficient condition of Theorem 1 , it is the optimal solution of problem (47).

\section{REFERENCES}

[1] K. Doppler, M. Rinne, C. Wijting, C. B. Ribeiro, and K. Hugl, "Deviceto-device communication as an underlay to LTE-advanced networks," IEEE Commun. Mag., vol. 47, no. 12, pp. 42-49, Dec. 2009.

[2] M. S. Corson, R. Laroia, J. Li, V. Park, T. Richardson, and G. Tsirtsis, "Toward proximity-aware internetworking," IEEE Wireless Commun., vol. 17 , no. 6, pp. 26-33, Dec. 2010.

[3] G. Fodor, E. Dahlman, G. Mildh, S. Parkvall, N. Reider, G. Miklos, and Z. Turanyi, "Design aspects of network assisted device-to device communications," IEEE Commun. Mag., vol. 50, no.3, pp. 170-177, Mar. 2012.

[4] T. D. Hoang, L. B. Le, and T. Le-Ngoc, "Joint subchannel and power allocation for D2D communications in cellular networks," in Proc. IEEE WCNC'2014, Apr. 2014.

[5] X. Lin, J. G. Andrews, A. Ghosh, and R. Ratasuk, "An overview on 3GPP device-to-device proximity services," IEEE Commun. Mag., vol. 52, no. 4, pp. 40-48, Apr. 2014.

[6] T. D. Hoang, L. B. Le, and T. Le-Ngoc, "Resource allocation for D2D communications under proportional fairness," in Proc. IEEE GLOBECOM'2014, Dec. 2014.

[7] E. Hossain, L. B. Le, and D. Niyato, Radio Resource Management in Multi-Tier Cellular Wireless Networks. New York, NY, USA: Wiley, 2013.

[8] Z. Hasan, H. Boostanimehr, and V. K. Bhargava, "Green cellular networks: A survey, some research issues and challenges," IEEE Commun. Surveys \& Tutorials, vol. 13, no. 4, pp. 524-540, Nov. 2011.

[9] G. Y. Li, Z. Xu, C. Xiong, C. Yang, S. Zhang, Y. Chen, and S. Xu, "Energy-efficient wireless communications: Tutorial, survey, and open issues," IEEE Wireless Commun., vol. 18, no. 6, pp. 28-35, Dec. 2011

[10] V. N. Ha and L. B. Le, "Fair resource allocation for OFDMA femtocell networks with macrocell protection," IEEE Trans. Veh. Technol., vol. 63 , no. 3, pp. 1388-1401.
[11] C. Xiong, G. Y. Li, S. Zhang, Y. Chen, and S. Xu, "Energy-efficient resource allocation in OFDMA networks," IEEE Trans. Commun., vol. 60, no. 12 , pp. $3767-3778$, Dec. 2012

[12] C. Isheden, Z. Chong, E. Jorswieck, and G. Fettweis, "Framework for link-Level energy efficiency optimization with informed transmitter," IEEE Trans. Wireless Commun., vol. 11, no. 8, pp. 2946-2957, Aug. 2012.

[13] S. Khakurel, L. Musavian, and T. Le-Ngoc, "Energy-efficient resource and power allocation for uplink multi-user OFDMA systems," in Proc. IEEE PIMRC'2012, pp. 357-361, Sept. 2012.

[14] M. Ismail, A. Gamage, W. Zhuang X. Shen, "Energy efficient uplink resource allocation in a heterogeneous wireless medium," in Proc. IEEE ICC'2014, pp. 5275-5280, Jun. 2014.

[15] C. H. Yu, O. Tirkkonen, K. Doppler, and C. Ribeiro, "On the performance of device-to-device underlay communication with simple power control," in Proc. IEEE VTC'2009, pp. 1-5, Apr. 2009.

[16] C. H. Yu, K. Doppler, C. B. Ribeiro, and O. Tirkkonen, "Resource sharing optimization for device-to-device communication underlaying cellular networks," IEEE Trans. Wireless Commun., vol. 10, no. 8, pp. 2752-2763, Aug. 2011.

[17] J. Wang, D. Zhu, C. Zhao, G. Y. Li, and M. Lei, "Resource sharing of underlaying device-to-device and uplink cellular communications," IEEE Commun. Lett., vol. 17, no. 6, pp. 1148-1151, Jun. 2013.

[18] D. Feng, L. Lu, Y. W. Yi, G. Y. Li, G. Geng, and S. Li, "Device-to-device communications underlaying cellular networks," IEEE Trans. Commun., vol. 61, no. 8, pp. 3541-3551, Aug. 2013.

[19] L. B. Le, "Fair resource allocation for device-to-device communications in wireless cellular networks," in Proc. IEEE Globecom'2012, pp. 54515456, Dec. 2012.

[20] Z. Zhou, M. Dong, K. Ota, J. Wu, and T. Sato, "Energy efficiency and spectral efficiency tradeoff in device-to-device (D2D) communications," IEEE Commun. Lett., vol. 3, no. 5, pp. 485-488, Oct. 2014.

[21] F. Wang, C. Xu, L. Song, Z. Han, and B. Zhang, "Energy-efficient radio resource and power allocation for device-to-device communication underlaying cellular networks," in Proc. IEEE WCSP'2012, pp. 1-6, Oct. 2012.

[22] D. Wu, J. Wang, R. Q. Hu, Y. Cai, and L. Zhou, "Energy-efficient resource sharing for mobile device-to-device multimedia communications," IEEE Trans. Veh. Technol., vol. 63, no. 5, pp. 2093-2103, Jun. 2014.

[23] T. D. Hoang, L. B. Le, and T. Le-Ngoc, "Dual decomposition method for energy-efficient resource allocation in D2D communications underlying cellular networks," in Proc. IEEE GLOBECOM'2015, Dec. 2015

[24] T. D. Hoang, L. B. Le, and T. Le-Ngoc, "Radio resource management for optimizing energy efficiency of D2D communications in cellular networks," in Proc. IEEE PIMRC'2015, Sep. 2015.

[25] W. O. Oduola, X. Li, L. Qian, and Z. Han, "Power control for deviceto-device communications as an underlay to cellular system," in Proc. IEEE ICC'2014, pp. 5257-5262, Jun. 2014.

[26] C. Gao, X. Sheng, J. Tang, W. Zhang, S. Zou, and G. Mohsen, "Joint mode selection, channel allocation and power assignment for green device-to-device communications," in Proc. IEEE ICC'2014, pp. 178183, Jun. 2014

[27] C. Xiong, G. Y. Li, S. Zhang, Y. Chen, and S. Xu, "Energy-efficient resource allocation in OFDMA networks," IEEE Trans. Wireless Commun., vol. 60, no. 12, pp. 3767-3778, Dec. 2012.

[28] T. D. Hoang, L. B. Le, and T. Le-Ngoc, "Energy-efficient resource allocation for D2D communications in cellular networks," in Proc. IEEE ICC'2015, Jun. 2015.

[29] G. Yu, L. Xu, D. Feng, R. Yin, G. Y. Li, and Y. Jiang, "Joint mode selection and resource allocation for device-to-device communications," IEEE Trans. Commun., vol. 63, no. 11, pp. 3814-3824, Nov. 2014

[30] J. P. G. Crouzeix and J. A. Ferland, "Algorithms for generalized fractional programming,"Mathematical Programming, vol. 52, pp. 191207, 1991

[31] W. Dinkelbach, "On nonlinear fractional programming," Management Science, vol. 13, pp. 492-498, Mar. 1967.

[32] Y. Xu, J. Wang, Q. Wu, A. Anpalagan, and Y.-D. Yao, "Opportunistic spectrum access in cognitive radio networks: Global optimization using local interaction games," IEEE J. Sel. Topics Signal Process., vol. 6, no. 2, pp. 180-194, Apr. 2012

[33] Y. Xu, J. Wang, Q. Wu, A. Anpalagan, and Y.-D. Yao, "Opportunistic spectrum access in unknown dynamic environment: A game-theoretic stochastic learning solution," IEEE Trans. Wireless Commun., vol. 11, no. 4, pp. $1380-1391$, Apr. 2012.

[34] S. Boyd and L. Vandenberghe, Convex optimization, Cambridge University Press, 2009. 
[35] D. P. Bertsekas, Nonlinear programming, 2nd ed. Cambridge, MA, USA: MIT Press, 2008.

[36] A. Nemirovski, Interior point polynomial methods in convex programming, Lecture notes, Georgia Inst. of Technol., Atlanta, GA, USA, 2004,

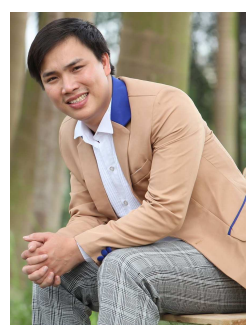

Tuong Duc Hoang (S'14) received the B.Eng. (Honor Program) degree from Hanoi University of Technology, Vietnam, in 2010, and the M.S. degree from Korea Advanced Institute of Science and Technology (KAIST), Daejeon, Korea, in 2013. He is currently a Ph.D. student at the Institut National de la Recherche Scientifique-Energy, Materials, and Telecommunications Center (INRS-EMT), Université du Québec, Montréal, QC, Canada. His research interest includes radio resource management for wireless communication systems with special emphasis on heterogeneous networks including D2D communications and dense networks.

Long Bao Le (S'04-M'07-SM'12) received the B.Eng. (with Highest Distinction) degree from Ho Chi Minh City University of Technology, Vietnam, in 1999, the M.Eng. degree from Asian Institute of Technology, Pathumthani, Thailand, in 2002, and the Ph.D. degree from the University of Manitoba, Winnipeg, MB, Canada, in 2007.

He was a postdoctoral researcher at Massachusetts Institute of Technology (2008-2010) and University of Waterloo (2007-2008). Since 2010, he has been an assistant professor with the Institut National de la Recherche Scientifique (INRS), Université du Québec, Montréal, QC, Canada. His current research interests include smartgrids, cognitive radio and dynamic spectrum sharing, radio resource management, network control and optimization for wireless networks. He is a co-author of the book Radio Resource Management in Multi-Tier Cellular Wireless Networks (Wiley, 2013). Dr. Le is a member of the editorial board of IEEE Communications Surveys and Tutorials and IEEE Wireless Communications Letters. He has served as a technical program committee chair/co-chair for several IEEE conferences including IEEE WCNC, IEEE VTC, and IEEE PIMRC. ch. 10 , p. 169.

[37] J.-B. Hiriart-Urruty and C. Lemarechal, Fundamental of Convex Analysis, Springer Verlag, Heigelbereg, 2001.

[38] A. H. Land, A. G. Doig, "An automatic method of solving discrete programming problems," Econometrica: Journal of the Econometric Society, vol. 28, no. 3, pp. 497-520, Jul. 1960.

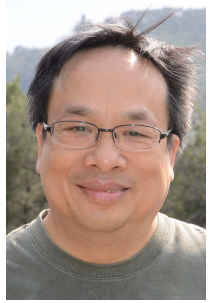

Tho Le-Ngoc (F'97) obtained his B.Eng. (with Distinction) in Electrical Engineering in 1976, his M.Eng. in 1978 from McGill University, Montreal, and his Ph.D. in Digital Communications in 1983 from the University of Ottawa, Canada. During 1977-1982, he was with Spar Aerospace Limited and involved in the development and design of satellite communications systems. During 1982-1985, he was an Engineering Manager of the Radio Group in the Department of Development Engineering of SRTelecom Inc., where he developed the new point-tomultipoint DA-TDMA/TDM Subscriber Radio System SR500. During 19852000, he was a Professor at the Department of Electrical and Computer Engineering of Concordia University. Since 2000, he has been with the Department of Electrical and Computer Engineering of McGill University. His research interest is in the area of broadband digital communications. He is a fellow of the Institute of Electrical and Electronics Engineers (IEEE), the Engineering Institute of Canada (EIC), the Canadian Academy of Engineering (CAE) and the Royal Society of Canada (RSC). He is the recipient of the 2004 Canadian Award in Telecommunications Research, and recipient of the IEEE Canada Fessenden Award 2005. He holds a Canada Research Chair (Tier I) on Broadband Access Communications. 